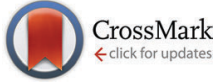

Cite this: Chem. Commun., 2016, 52,9327

Received 25th April 2016, Accepted 7th June 2016

DOI: $10.1039 / c 6 c c 03439 j$

www.rsc.org/chemcomm

\section{Cyclic dinucleotide (c-di-GMP, c-di-AMP, and cGAMP) signalings have come of age to be inhibited by small molecules}

\author{
Clement Opoku-Temeng, ${ }^{\text {ab }}$ Jie Zhou, ${ }^{a}$ Yue Zheng, ${ }^{\text {ab }}$ Jianmei Su $^{\mathrm{b}}$ and \\ Herman O. Sintim*a
}

\begin{abstract}
Bacteria utilize nucleotide-based second messengers to regulate a myriad of physiological processes. Cyclic dinucleotides have emerged as central regulators of bacterial physiology, controlling processes ranging from cell wall homeostasis to virulence production, and so far over thousands of manuscripts have provided biological insights into c-di-NMP signaling. The development of small molecule inhibitors of c-di-NMP signaling has significantly lagged behind. Recent developments in assays that allow for high-throughput screening of inhibitors suggest that the time is right for a concerted effort to identify inhibitors of these fascinating second messengers. Herein, we review c-di-NMP signaling and small molecules that have been developed to inhibit cyclic dinucleotide-related enzymes.
\end{abstract}

\section{Introduction}

The ability of cells to sense and respond to environmental cues is critical for survival. Signals such as nutrition, stress, changes in temperature, osmolarity and $\mathrm{pH}$ must be precisely sensed and adequately responded to. Second messengers are crucial in the transduction of signals and nucleotide-based second messengers have over the years been demonstrated to be central to this process (Fig. 1). ${ }^{1}$ Cyclic mononucleotides, such as cAMP and cGMP (Fig. 2), are key second messengers in both bacteria and higher organisms. For example, cAMP controls carbon metabolism, motility and virulence. ${ }^{2}$ cGMP has also been shown to control a variety of key biological processes, such as smooth muscle relaxation and regulation of the intestinal electrolyte and fluid homeostasis in mammals. ${ }^{3}$ In bacteria, cGMP has been shown to regulate the development of Rhodospirillum centenum, an alphaproteobacterium. ${ }^{4}$ The alarmones, guanosine tetraphosphate and guanosine pentaphosphate ((p)ppGpp), have been shown to regulate stringent response in bacteria. ${ }^{5,6}$ Nicotinic acid adenine dinucleotide phosphate (NAADP), adenosine diphosphoribose (ADPR), and cyclic ADP-ribose (cADPR) function as $\mathrm{Ca}^{2+}$ mobilizers that affect various cellular processes. ${ }^{7}$ The cADPR analog, cyclic inosine diphosphoribose ether (cIDPRE), has also been shown to cause $\mathrm{Ca}^{2+}$ release in human Jurkat T-lymphocytes. ${ }^{8}$ Dinucleotides, such as NAD and NADP, are also important

\footnotetext{
${ }^{a}$ Department of Chemistry, Center for Drug Discovery, Purdue University, West Lafayette, IN 47907, USA. E-mail: hsintim@purdue.edu; Tel: +1 (765) 494-5200

${ }^{b}$ Graduate Program in Biochemistry, University of Maryland, College Park,

Maryland 20742, USA
}

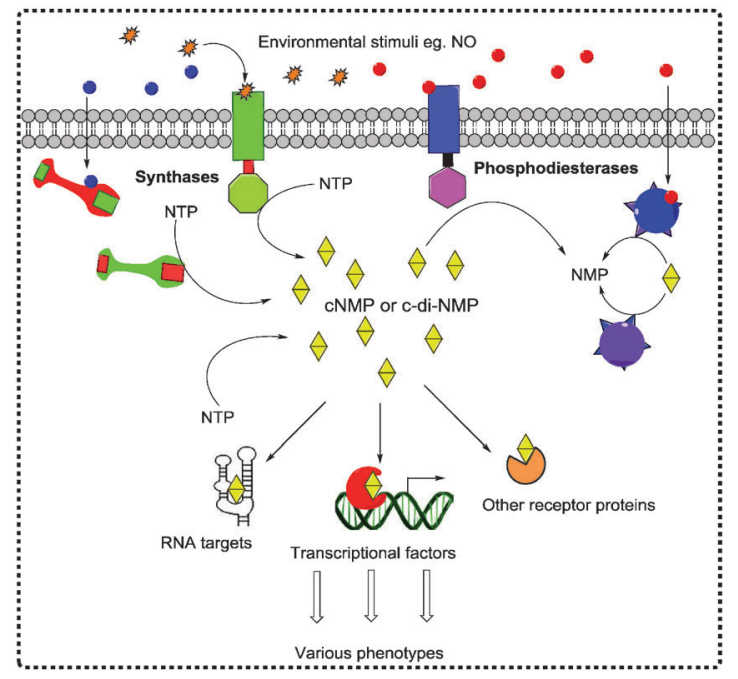

B.

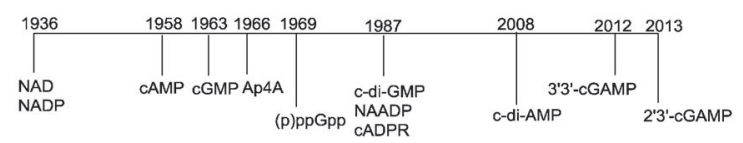

Fig. 1 (A) Signal transduction from a primary signal (first messengers) to second messengers that relay and amplify information to macromolecule regulation that affects the phenotype. (B) Timeline of the discovery of nucleotide-based second messengers.

signaling molecules and are involved in T-cell calcium signaling. ${ }^{7}$ Diadenosine polyphosphates (Ap2A, Ap3A, Ap4A, Ap5A and Ap6A) 

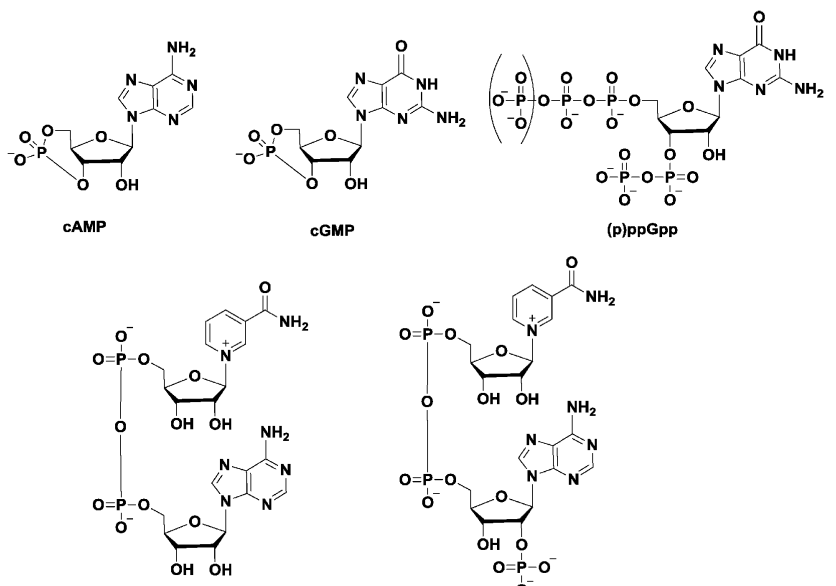

GMP

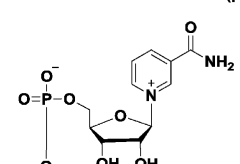

)ppGpp

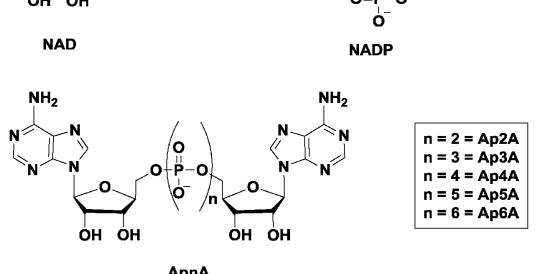

Fig. 2 Structures of some of the well-established nucleotide second messengers.

are also ubiquitous second messengers with physiological roles ranging from serving as alarmones to being vasoactive molecules. ${ }^{9-11}$ Fig. 2 shows the structures of some of the wellestablished nucleotide second messengers.

Due to the importance of nucleotide second messengers in biology, massive efforts have been focused on the development of small molecules that inhibit the signaling of these second messengers. The majority of inhibitor developments against nucleotide second messengers had focused on cAMP/cGMP and several small molecules that inhibit the phosphodiesterases of cyclic nucleotide monophosphate are now used in the clinic (see Fig. 3 for examples).

Cyclic dinucleotides (see Fig. 4 for structures) have now emerged as important second messengers and the dazzling arrays of processes that these molecules appear to regulate suggest that these dinucleotides will also become as important as cyclic nucleotide monophosphates. ${ }^{1,12-19}$ The first cyclic dinucleotide,

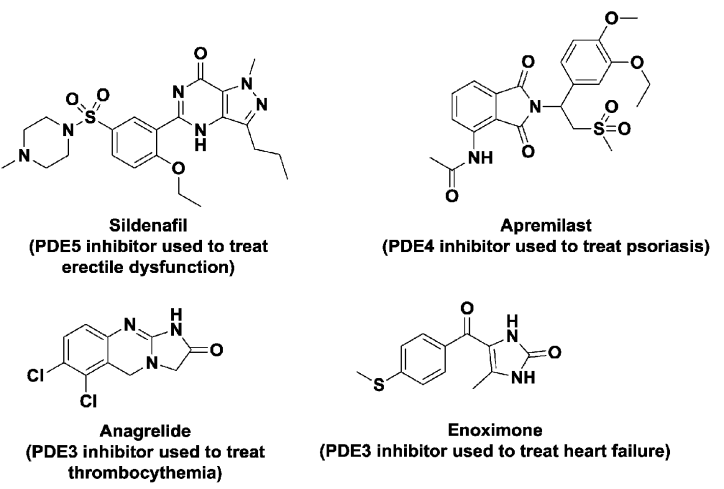

Fig. 3 Structures of some PDE inhibitors and their clinical uses.

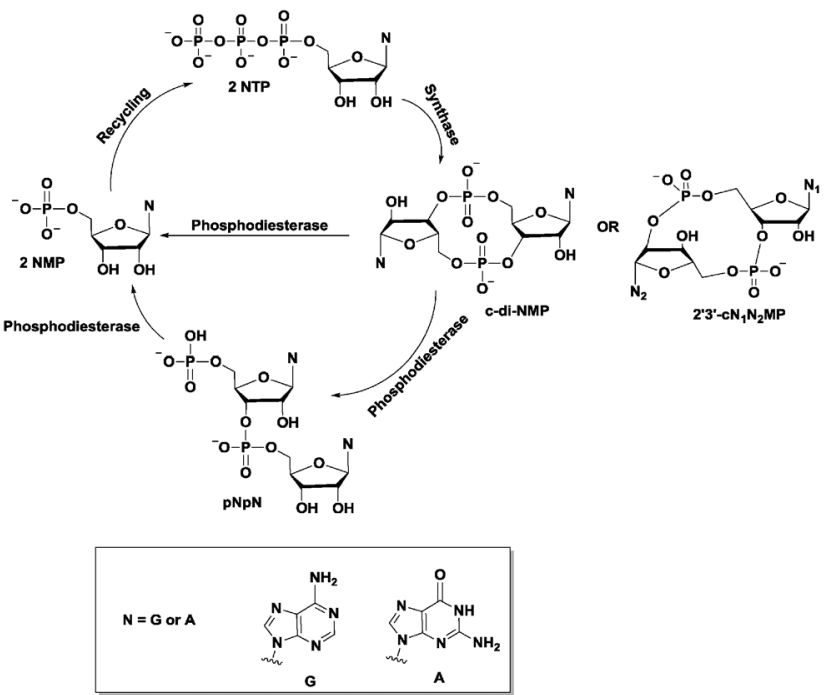

Fig. 4 Schematic of the general synthesis and degradation of cyclic dinucleotide second messengers.

c-di-GMP, was the first to be discovered by Benziman in 1987 and has since been established as the master regulator of the bacterial lifestyle in mostly Gram-negative and a handful of Gram-positive bacteria. ${ }^{1,20}$ c-di-AMP was also later identified to be a crucial second messenger in Gram-positive bacteria and mycobacteria. ${ }^{1,21}$ Subsequently, hybrid cyclic dinucleotides cGAMP $\left(2^{\prime} 3^{\prime} \text { and } 3^{\prime} 3^{\prime}\right)^{22,23}$ were also identified to regulate various processes. $^{24,25}$ Efforts towards understanding the molecular mechanisms underlying the activities of cyclic dinucleotides have uncovered a myriad of cyclic dinucleotide synthases, phosphodiesterases, effector proteins and RNA, all of which work in a concerted manner to execute a specific action in response to the cyclic dinucleotide second messengers. ${ }^{13,21,26}$

Due to the seemingly indispensable roles of cyclic dinucleotides in both bacteria and higher organisms (in this case as immune response regulators), research has also been directed towards ways of interrupting cyclic dinucleotide signaling. Consequently, various inhibitors have been discovered/developed against cyclic dinucleotide signaling. The field of cyclic dinucleotide inhibitor discovery is now in its infancy with only a handful of groups pioneering inhibitor development but it is projected that there will be an explosion of research activities towards the development of small molecule regulators of cyclic dinucleotide signaling as it becomes increasingly clear that these second messengers are critical for various biological processes and diseased states. In this review, we discuss some of the fundamentals of cyclic dinucleotide signaling with emphasis on inhibitors developed against the cyclic dinucleotides.

\section{c-di-GMP}

Bis- $\left(3^{\prime}-5^{\prime}\right)$ cyclic diguanylic acid (c-di-GMP) was originally discovered by Benziman and colleagues in 1987 as an activator of cellulose synthase in a Gram-negative bacterium, Gluconoacetobacter xylinus (formerly named Acetobacter xylinum). ${ }^{20}$ Although it was discovered almost 30 years ago, it is only in 
the last decade that the many cellular processes that are regulated by c-di-GMP have been elucidated. The major function of c-di-GMP is the regulation of motility-to-sessility transition. ${ }^{1}$ It is also a key player in the cell cycle and virulence factor production. ${ }^{1}$ So far, c-di-GMP has been identified in a wide variety of Gram-negative bacteria, including Pseudomonas aeruginosa, Caulobacter crescentus, Escherichia coli, Salmonella typhimurium and Vibrio cholerae, and a few Gram-positive bacteria, such as Bacillus subtilis and Listeria monocytogenes. ${ }^{1}$

\section{c-di-GMP metabolism enzymes}

Since c-di-GMP plays important roles in the cell, there is interest in modulating the intracellular concentrations of the dinucleotide with small molecules. The intracellular concentration of c-di-GMP is fine-tuned by its metabolism enzymes, diguanylate cyclase (DGC) and phosphodiesterase (PDE) (Fig. 4-7). DGCs cyclize two molecules of GTP to c-di-GMP and release two molecules of pyrophosphates. DGC was first purified and tested in vitro by the Benziman group. ${ }^{20}$ In 1995 , Newton and colleagues characterized the gene of a DGC PleD in Caulobacter crescentus and identified a novel C-terminal GGDEF (Gly-Gly-Asp-Glu-Phe) domain. ${ }^{27}$ The conserved GGDEF or GGEEF (Gly-Gly-Glu-Glu-Phe) motifs were confirmed to be commonly shared by most DGCs. ${ }^{28-30}$ Some well characterized examples are $\mathrm{WspR}^{29}$ from P. aeruginosa (PDB 3I5A) and DosC ${ }^{31}$ (also known as YddV) from E. coli (PDB 4ZVE). Some non-canonical domains also display DGC functions, such as the AGDEF domain in $V$. cholerae VCA0965 $5^{32}$ and the SGDEF domain in Pectobacterium atrosepticum ECA3270. ${ }^{33}$ Besides the active site (A-site), some DGCs also contain
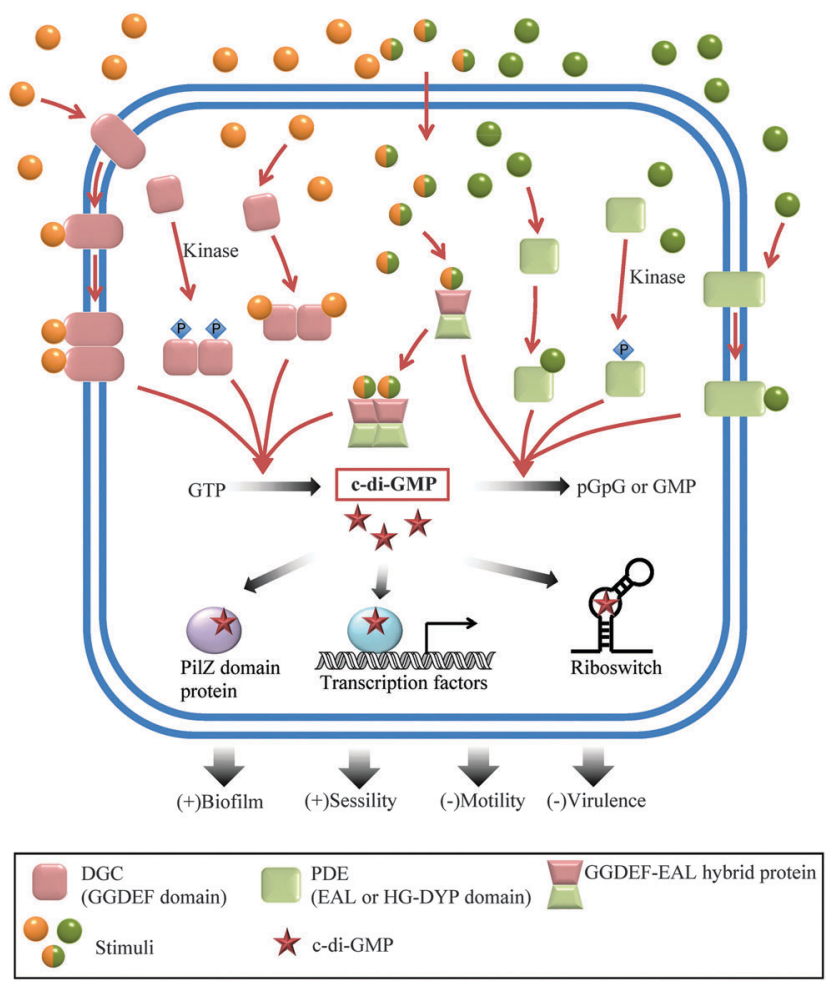

Fig. 5 Overview of c-di-GMP regulation in bacteria. an inhibitory site (I-site). When c-di-GMP binds to the I-site of the DGC, characterized by the $\operatorname{RxxD}$ ( $\mathrm{x}$ refers to any amino acid) sequence, c-di-GMP synthesis is allosterically inhibited. ${ }^{34}$

The hydrolysis of c-di-GMP is conducted by two types of phosphodiesterases (PDEs): EAL (Glu-Ala-Leu) domain PDE and HD-GYP (His-Asp and Gly-Tyr-Pro) domain PDE (Fig. 2 and 5). The major product of EAL domain PDE is $5^{\prime}$-phosphoguanylylguanosine (pGpG) and the degradation of $\mathrm{pGpG}$ to guanosine monophosphate (GMP) is slow. For example, RocR (PDB 3SY8) ${ }^{36}$ from $P$. aeruginosa cleaves c-di-GMP into $\mathrm{pGpG}$ in the presence of $\mathrm{Mg}^{2+}$ or $\mathrm{Mn}^{2+}$ and this reaction is inhibited by $\mathrm{Ca}^{2+}$ and $\mathrm{Zn}^{2+}$ ions. $^{37}$ Recently it was disclosed that in bacteria, such as $P$. aeruginosa, which harbour PDEs that mainly cleave c-di-GMP into pGpG, a second PDE (an oligoribonuclease) completes the cleavage of pGpG into GMP. ${ }^{38,39}$ The role of oligoribonuclease in cyclic dinucleotide metabolism is expected because these enzymes (found in several bacteria) have long been known to cleave short oligonucleotides into NMPs. ${ }^{40-42}$ Galperin and colleagues predicted the functions of HD-GYP in c-di-GMP hydrolysis by bioinformatic studies ${ }^{43}$ and the biochemical evidence for this hypothesis was shown by Dow and colleagues. ${ }^{44}$ The HD-GYP domain PDE RpfG from Xanthomonas campestris pv. campestris directly cleaves c-di-GMP into GMP. ${ }^{44}$ There are a few crystal structures of HD-GYP PDEs reported. Bd1817 from Bdellovibrio bacteriovorus (PDE 3TM8), which lacks the tyrosine in the GYP active site, presents a bi-iron center. ${ }^{45}$ However, the structure of another HD-GYP protein PmGH from Persephonella marina shows a different trinuclear iron active site (PDB 4ME4). ${ }^{46}$ In 2015, the structure of PA4781 from Pseudomonas aeruginosa was reported (PDB 4R8Z) and the bimetallic active site binds to $\mathrm{Mn}^{2+}, \mathrm{Ni}^{2+}$ and some other transition metals with similar affinities. ${ }^{47}$

\section{c-di-GMP receptors}

Different types of c-di-GMP binding proteins have been identified, augmenting the important roles played by c-di-GMP in the bacterial lifestyle (planktonic or sessile). ${ }^{13}$ When the intracellular concentration of c-di-GMP is high, it induces extracellular polymeric substance (EPS) production and promotes biofilm formation. ${ }^{48}$ When c-di-GMP concentration is low, flagella biosynthesis is upregulated and bacterial motility significantly increases. $^{49}$ c-di-GMP drives the $C$. crescentus cell cycle by inducing the dephosphorylation or degradation of a replication initiation inhibitor CtrA through the effector proteins CckA and PopA respectively. ${ }^{50,51}$ c-di-GMP also represses acute virulence gene transcription. ${ }^{52}$ For some intracellular pathogens, c-di-GMP has been shown to make bacterial cell surface proteins resistant to proteolytic cleavage, enhancing bacterial invasion. ${ }^{53}$ There are three major types of c-di-GMP binding proteins: (1) the Pilz domain c-di-GMP receptor; (2) DGC I-sites and inactive EAL and HD-GYP domains; and (3) other types of c-di-GMP receptors. ${ }^{13}$

The first type of c-di-GMP binding domain PilZ (Pfam: PF07238) was predicted by Amikam and Galperin by bioinformatics studies. ${ }^{54}$ This hypothesis was verified by Gomelsky and colleagues in the same year. ${ }^{55}$ The purified PilZ domain protein YcgR from E. coli showed a dissociation constant $\left(K_{\mathrm{d}}\right)$ of $0.84 \pm 0.16 \mu \mathrm{M}$ to 


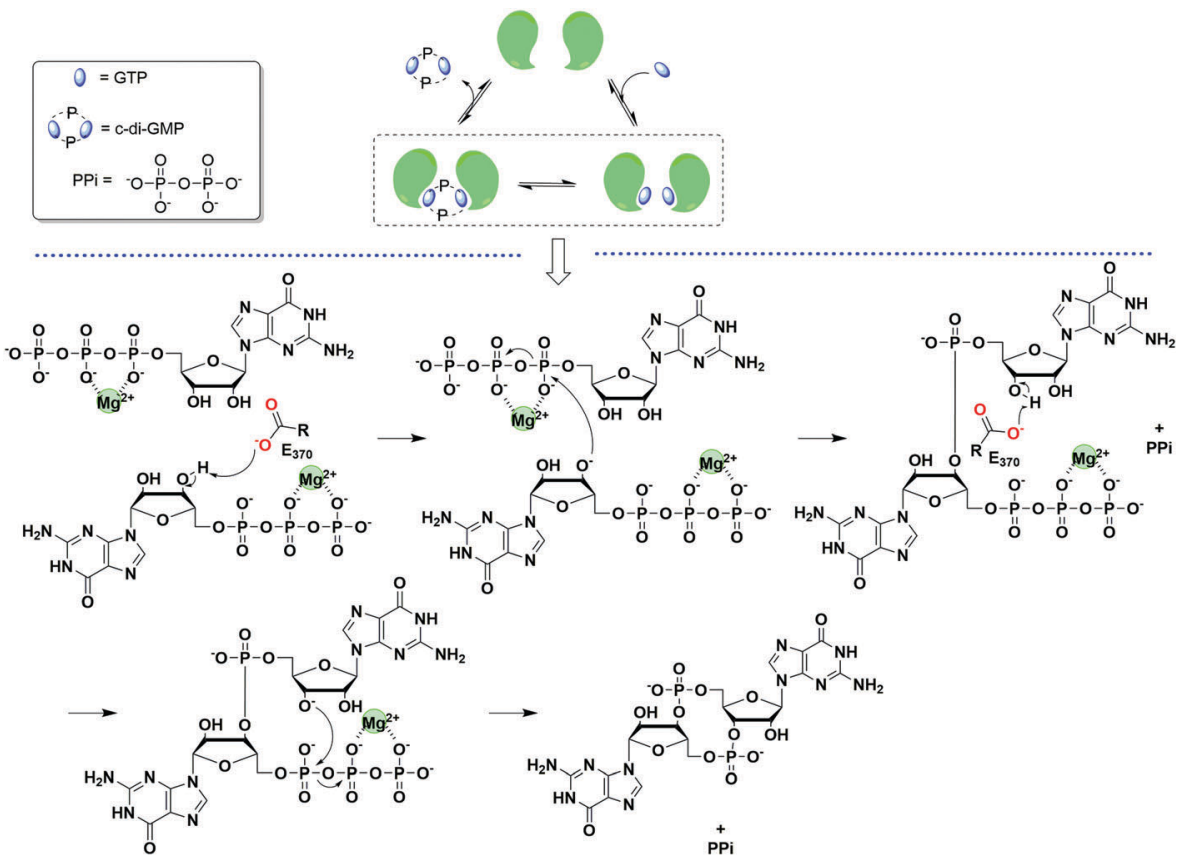

Fig. 6 General scheme of the mechanism of c-di-GMP synthesis. The example was taken from PleD. ${ }^{35}$ (PDB 1W25).

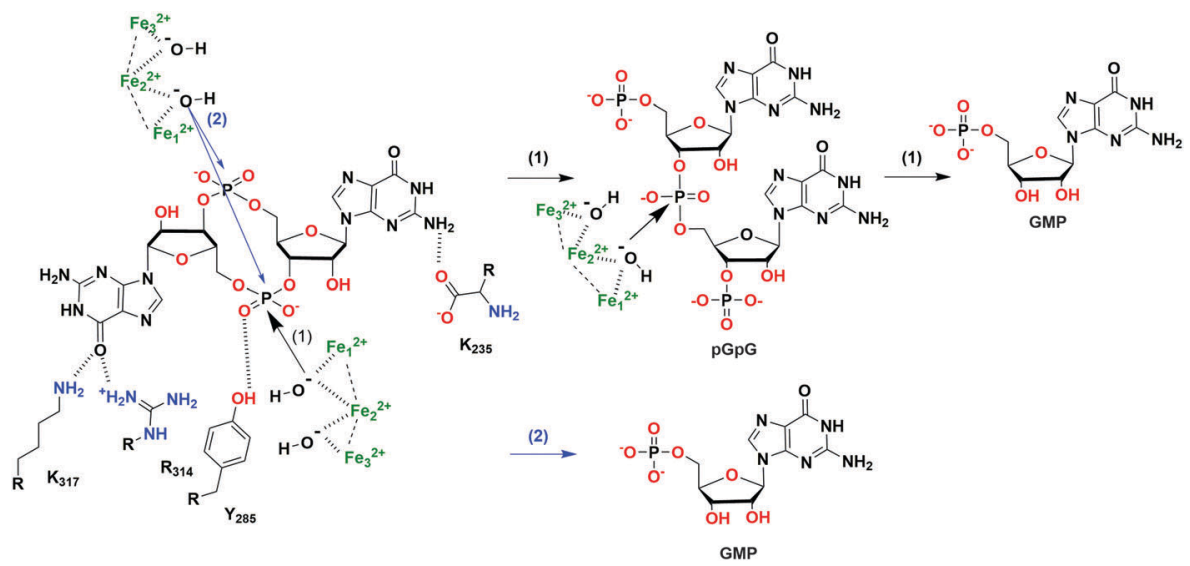

Fig. 7 The hydrolysis mechanism of c-di-GMP by the HD-GYP domain protein from Persephonella marina (PmGH). ${ }^{46}$ (PDB 4MDZ, PmGH in complex with c-di-GMP). Scheme (1) (black arrows) indicates a two-step hydrolysis of c-di-GMP, an attack by one activated water molecule on one phosphodiester linkage forms pGpG which is further degraded into GMP; scheme (2) (blue arrows) indicates a one-step hydrolysis of c-di-GMP, an attack by two activated water molecules on both phosphodiester linkages directly forms GMP.

c-di-GMP and the $\mathrm{C}$ terminus of BcsA from G. xylinus also binds to c-di-GMP. ${ }^{55}$ The Pilz domain is widely spread in many bacteria. Alg44 from $P$. aeruginosa ${ }^{56}$ DgrA protein from C. crescentus, ${ }^{57}$ and $\mathrm{PlzC}$ and PlzD from $V$. cholerae ${ }^{58}$ are typical Pilz domain c-di-GMP receptors. The conserved sequence $\operatorname{RxxxR}_{20-30}(\mathrm{D} / \mathrm{N}) \mathrm{x}(\mathrm{S} / \mathrm{A}) \mathrm{xxG}$ ( $x$ refers to any amino acid) in the Pilz domain, which is responsible for c-di-GMP binding, was disclosed by crystal structures and biochemical experiments. ${ }^{13,55,56}$

As stated earlier, DGC activity is allosterically inhibited when c-di-GMP binds to the I-site. c-di-GMP strongly inhibits PleD from $C$. crescentus with a $K_{\mathrm{i}}$ of $0.5 \mu \mathrm{M} .^{59}$ A protein with degenerate GGDEF or EAL domains is another kind of c-di-GMP receptor. For example, the GGDEF-EAL domain FimX from
P. aeruginosa has neither DGC nor PDE activity (PDB 3HV8). ${ }^{60}$ Its EAL domain binds to c-di-GMP and regulates twitching motility. ${ }^{60}$ Another example is the GGDEF domain of PopA from $C$. crescentus, which does not synthesize c-di-GMP but rather mediates movement toward the cell pole. ${ }^{61}$

Some c-di-GMP receptors are transcription factors that up-regulate or down-regulate target gene transcription upon c-di-GMP binding, such as the major flagella gene regulator in P. aeruginosa FleQ (PDB $4 \mathrm{WXM}$ ). ${ }^{62}$ c-di-GMP binds to the Walker A motif of FleQ resulting in decreased flagella biosynthesis and increased EPS production. ${ }^{63}$ Furthermore, binding of c-di-GMP to VpsT, a transcription factor in $V$. cholerae, causes the dimerization of VpsT thereby enhancing its role in regulating biofilm formation. ${ }^{64}$ 
In 2008, Breaker and colleagues reported the first type of c-di-GMP riboswitch, Vc2 RNA. ${ }^{65}$ The riboswitch was observed to change conformation upon c-di-GMP binding. This facilitates the transcriptional regulation of downstream genes by the Vc2 riboswitch. ${ }^{65}$ The same group, in 2010, identified the c-di-GMP-II riboswitch which had picomolar affinity for c-di-GMP and was found to regulate self-splicing. ${ }^{66}$

\section{Polymorphism of c-di-GMP}

c-di-GMP has the tendency to readily form dimers, tetraplexes and higher aggregates in the presence of cations, not only in solution, ${ }^{67}$ but also when bound to proteins (see Fig. 8). Subtle changes in c-di-GMP can lead to a dramatic change in the propensity to form such higher aggregates. ${ }^{68}$ When developing c-di-GMP-based small molecule inhibitors against the various proteins and RNAs that bind to c-di-GMP, it is important to consider how any changes to the native c-di-GMP would affect the conformer and/or polyphorphism of the analog. ${ }^{68-70}$

\section{Assays for the discovery of c-di-GMP inhibitors}

Due to the importance of c-di-GMP signaling, there has been interest in developing small molecules to interrupt signaling. Towards this goal, several groups have developed assays that are amenable to high throughput screening inhibitor discovery. Following the discovery of c-di-GMP riboswitches that potentially bind to c-di-GMP, Sintim and colleagues reported a hybrid Spinach-Vc2 RNA aptamer, which couples the binding of c-di-GMP to the fluorescence of DFHBI, ${ }^{73}$ and this strategy has been utilized by others to develop riboswitch-based sensors that detect cyclic dinucleotides in complex environments,

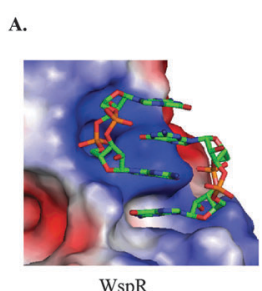

WspR

D.
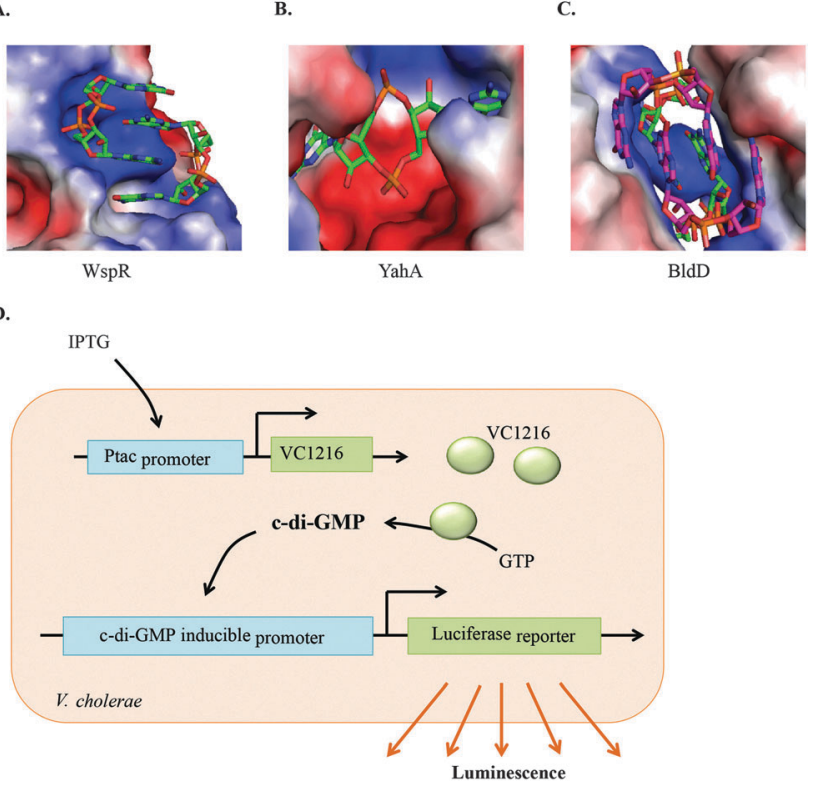

ig. 8 Polymorphism of c-di-GMP when bound to $(A)$ the I-site of WspR, a DGC (PDB 315A), (B) YahA, PDE (PDB 4LJ3) and (C) BldD, a transcriptional factor (PDB 4OAZ). (D) Detection of $c$-di-GMP by $V$. cholerae luminescence. $^{71}(\mathrm{~A}),(\mathrm{B})$, and (C) Reproduced with permission from ref. 72. Copyright (C) 2016 Future Science. including inside bacterial cells. ${ }^{74-77}$ The riboswitch sensor, albeit being sensitive, is too expensive to use in high throughput screenings for inhibitor discovery. The Sintim group has demonstrated that intercalators that fluoresce or have fluorescence being quenched upon binding to c-di-GMP could be used as a simple means to screen for inhibitors of c-di-GMP processing enzymes. One example is that c-di-GMP can aggregate into supremolecular structures in the presence of thiazole orange (TO), leading to fluorescence turn-on. ${ }^{78}$ In another example, the same group demonstrated that c-di-GMP/proflavine association leads to fluorescence turn-off. ${ }^{79}$ A fluorescent c-di-NMP sensor that incorporates the aminopurine nucleobase and reports on PDE activity has also been reported. ${ }^{80}$ Such fluorescence assays could be used to screen for inhibitors of c-di-GMP synthesis or degradation. Others have used computational virtual screening ${ }^{81}$ and differential radial capillary action of ligand assay (DRaCALA) ${ }^{82}$ to identify c-di-GMP inhibitors. Perhaps a better way to discover c-di-GMP signaling inhibitors that are cell permeable is to use cellbased systems. Waters and colleagues have developed a $V$. cholerae bioluminescent reporter strain that can be used to screen for DGC inhibitors $^{71}$ (see Fig. 8D). The reporter strain contains a plasmid harboring an IPTG-inducible DGC gene, VC1216. When treated with IPTG, VC1216 expression was induced with a corresponding increase in c-di-GMP production. The reporter strain also contains a second plasmid that encodes a luciferase operon under the control of a c-di-GMP inducible promoter. Any compound that inhibits VC1216 activity would lead to a change in bacterial luminescence. Such reporter strains or variations thereof are going to facilitate the discovery of cellpermeable c-di-GMP inhibitors.

\section{Inhibitors of c-di-GMP}

The redundancy of GGDEF, EAL and HD-GYP domains in bacteria, which are typical domains of c-di-GMP DGCs and PDEs pose challenges for the development of small molecules to modulate c-di-GMP signaling in bacteria. ${ }^{83}$ Table 1 shows the distribution of c-di-GMP DGCs and PDEs in select bacteria. c-di-GMP regulation by DGCs and PDEs is complicated at both the global level and the local level. Some DGCs and PDEs contribute to the total c-di-GMP concentration, but others only affect c-di-GMP concentration in a localized region. ${ }^{52}$ Römling and colleagues showed that in Salmonella, a mutation of the major DGC AdrA leads to a significant decrease in the global level of c-di-GMP. ${ }^{84}$ The expression of the biofilm regulator CsgD was not affected due to localized pools of c-di-GMP from two other DGCs STM2123 and STM3388. ${ }^{84}$ Some DGCs or PDEs regulate bacterial phenotypes without changing the overall c-di-GMP concentration. In Pseudomonas aeruginosa, mutating the $n b d A$ gene which encodes the c-di-GMP PDE NbdA had no effect on intracellular c-di-GMP levels. ${ }^{85}$ However, the mutant strain was found to be resistant to biofilm dispersion by nitric oxide (NO) relative to the wildtype. ${ }^{85}$ Therefore a major challenge in inhibitor development is finding small molecules that can either inhibit all of these redundant enzymes or selectively inhibit one over the other.

So far, efforts to develop c-di-GMP signaling inhibitors have mainly been focused on finding inhibitors to interfere with 
Table 1 Distribution of DGCs and PDEs of c-di-GMP in select bacteria ${ }^{83}$

\begin{tabular}{lccl}
\hline Species & GGDEF & EAL & HD-GYP \\
\hline Mesorhizobium loti & 32 & 18 & 1 \\
Pseudomonas aeruginosa & 33 & 21 & 3 \\
Escherichia coli & 19 & 17 & 0 \\
Bacillus subtilis & 4 & 3 & 0 \\
Bacillus halodurans & 4 & 2 & 2 \\
Mycobacterium tuberculosis & 1 & 2 & 0 \\
Vibrio cholerae & 41 & 22 & 9 \\
Caulobacter crescentus & 11 & 10 & 0 \\
Synechocystis sp. & 23 & 13 & 2 \\
Mycobacterium leprae & 3 & 2 & 0 \\
Xylella fastidiosa & 3 & 3 & 1 \\
Deinococcus radiodurans & 16 & 5 & 4 \\
Aquifex aeolicus & 11 & 8 & 1
\end{tabular}

c-di-GMP synthase DGC, ${ }^{82,86-93}$ PDE (EAL domain) ${ }^{94,95}$ and riboswitches. ${ }^{96,97}$ This is mostly because of the phenotypes that have been observed upon deletion of either DGC, PDE or their interacting partners. O'Toole and colleagues showed that deletion of the $P$. aeruginosa PDE, BifA, resulted in altered swarming mobility, EPS production and biofilm formation ${ }^{98}$ (Fig. 9). Also deletion of WspF, which caused the constitutive activation of the $P$. aeruginosa DGC WspR resulted in a strain with increased biofilm forming ability ${ }^{99}$ (Fig. 9). In a murine acute infection model, $P$. aeruginosa PA14 strains with mutation in the rocR gene were not virulent, evident by the survival of mice 5 days after infection ${ }^{100}$ (Fig. 9).

Table 2 and Fig. 10 depict the list of c-di-GMP inhibitors and their structures respectively. The first two examples of DGC inhibitors, glycosylated triterpenoid saponins (GTSs) and papulacandin B, were identified by Benziman and co-workers. ${ }^{93}$ However, such molecules probably lack the ability to cross bacterial membranes, making them not drug-like. Waters and co-workers reported several other small molecules, including $N$-(4-anilinophenyl)benzamide and LP-3145 as potential DGC inhibitors, to prevent biofilm formation in $V$. cholerae and P. aeruginosa respectively. ${ }^{87,88}$ Also, Landini and co-workers found that sulfathiazole and azathioprine inhibited biofilm formation by an $E$. coli strain harbouring $P$. aeruginosa DGC, WspR. ${ }^{89}$ The real mechanism is not known yet, however, sulfathiazole and azathioprine may affect the biosynthesis of c-di-GMP because of their antimetabolite activities, rather than directly inhibiting the activity of DGC..$^{89,90}$ Of note, azathioprine is used as an anti-inflammatory drug in the treatment of Crohn's disease and rheumatoid arthritis. ${ }^{101}$ Therefore, its toxicity profile has already been established and could become a safe antibiofilm drug for clinical applications. Recently, Lee and co-workers reported that ebselen inhibits WspR via covalent modification of a cysteine group near the active site. ${ }^{82}$ Ebselen is an alkylating agent as well as a planar aromatic molecule. It is also a promiscuous inhibitor and has been reported to inhibit many other biological targets ${ }^{102,103}$ and might not be an ideal drug candidate due to its potential toxicity. ${ }^{82}$ Others have also identified c-di-GMP signaling inhibitors via in silico screening. Rinaldo and co-workers identified catechol-containing sulfonohydrazide as an inhibitor of PleD with $\mathrm{IC}_{50}$ around $11 \mu \mathrm{M} .{ }^{81}$

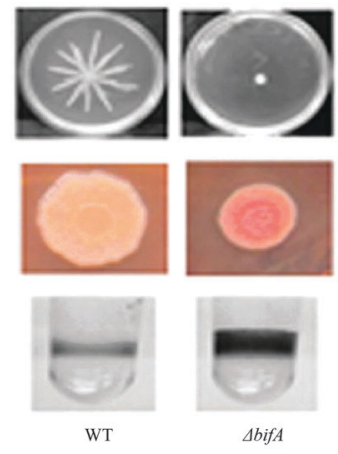

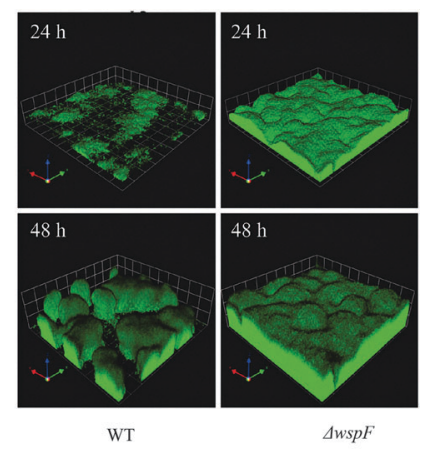

C.

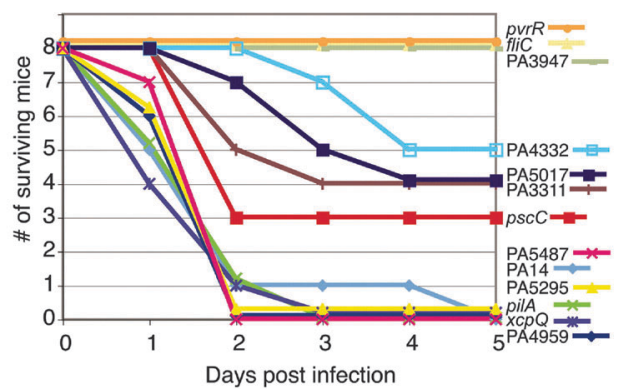

Fig. 9 (A) The effects of c-di-GMP PDE BifA on swarming motility, EPS production and biofilm formation. BifA mutant attenuated swarming motility. Red, wrinkly colony on congo red plates indicated increased EPS production. BifA mutant strain biofilm formation was increased compared to WT strain. (B) WspF mutation resulted in the activation of WspR, a c-di-GMP DGC. $\Delta$ wspF strain produced a thicker biofilm than WT strain. (C) Survival of mice post infection with wildtype $P$. aeruginosa PA14 and strains with various mutations in the DGC, PDE and DGC-PDE genes. Strains with PA3947 gene (RocR) mutation are less virulent. (A) Reproduced with permission from ref. 98. Copyright (C) 2010 American Society for Microbiology. (B) Reproduced with permission from ref. 99. Copyright (C) 2005, The National Academy of Sciences of the USA. (C) Reproduced with permission from ref. 100. Copyright (C) 2006, The National Academy of Sciences of the USA.

From the foregoing, it is clear that the development of cell permeable inhibitors of c-di-GMP signaling is only at its infancy and many of the reported inhibitors do have various limitations that will prevent clinical development.

A number of nucleotide-based c-di-NMP analogs have been designed and synthesized in order to differentially inhibit different receptors, including backbone and sugar modifications of c-di-GMP ${ }^{53,68,92,94,104}$ and neutral analogs of c-di-GMP. ${ }^{105-108}$ of note, endo-S c-di-GMP was shown to selectively inhibit c-di-GMP PDE but not DGC ${ }^{68}$ while $2^{\prime}$-F-c-di-GMP could inhibit DGCs better (4 times) than the native dinucleotide, c-di-GMP. ${ }^{92}$ Recently, Rinaldo and co-workers reported the first neutral c-di-GMP-like molecules for discriminating DGCs and PDEs, simply by replacing a phosphodiester bond with triazole. ${ }^{108}$ Potential limitations of c-di-GMP analogs as clinical candidates are solubility issues (guanine containing molecules tend to aggregate) and lack of cell permeation (most bisguanine-based analogs have too many hydrogen bond acceptor and donor units in the molecule and hence are not drug-like). 
Table 2 Summary of small molecules identified as c-di-GMP inhibitors

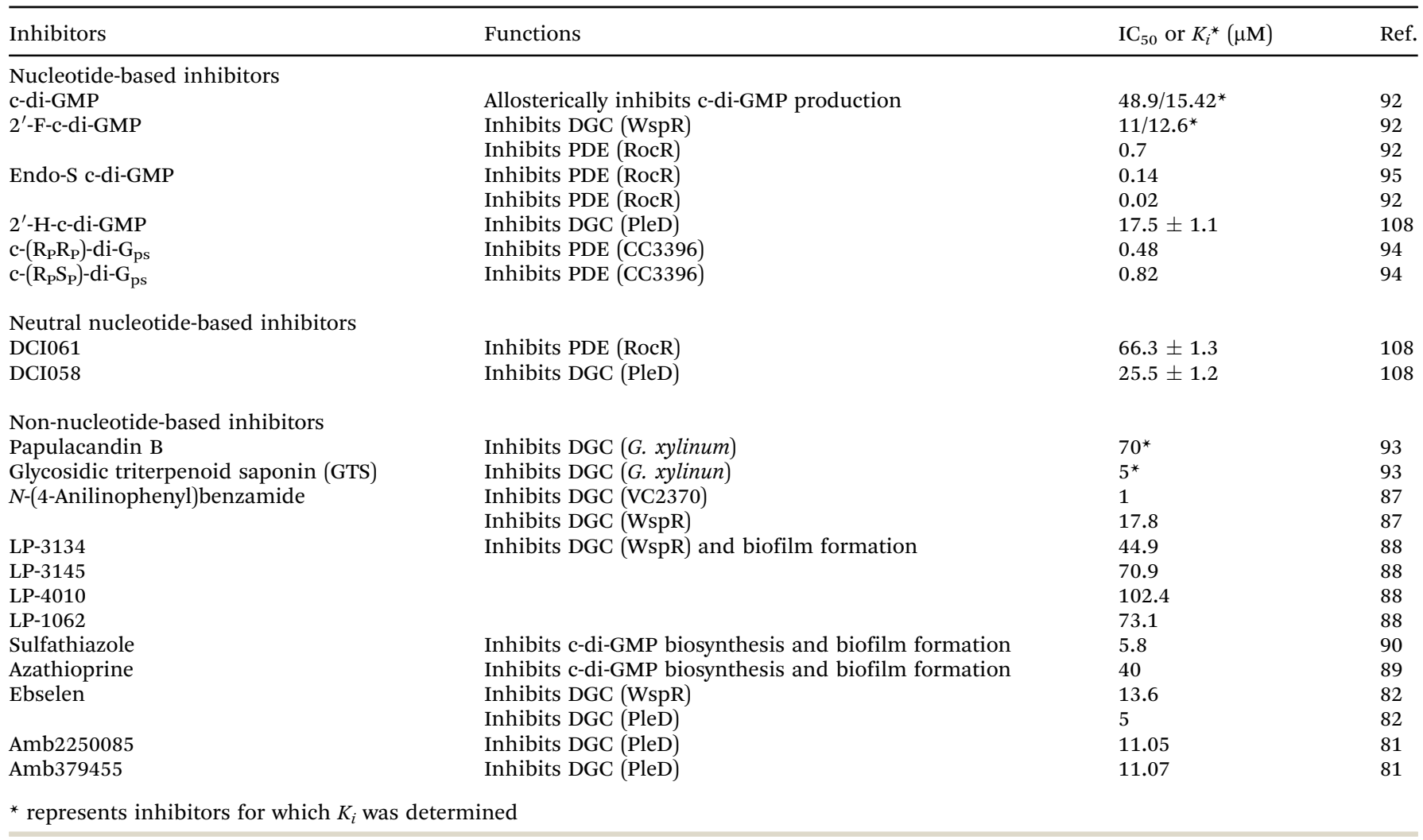

Recently, Sintim and co-workers have suggested an interesting strategy to inhibit c-di-GMP and c-di-AMP signaling via dinucleotide aggregation with small molecules (Fig. 11). ${ }^{72}$ Although only modest inhibitions of c-di-GMP/AMP degradation by PDEs were observed by this strategy, it is encouraging that one could bypass the enzyme redundancy issue that makes the global inhibition of c-di-NMP in bacteria challenging by targeting the dinucleotide itself. $^{72}$

\section{c-di-AMP}

B. subtilis DNA integrity scanning protein A (DisA) is a protein involved in maintaining DNA integrity at the onset of $B$. subtilis sporulation. ${ }^{109,110}$ In the course of structural characterization of DisA from Thermatoga maritima, Hopfner and colleagues in 2008 discovered bis- $\left(3^{\prime}-5^{\prime}\right)$ cyclic diadenylic acid (c-di-AMP) bound to the nucleotide binding site of DisA. ${ }^{109}$ The authors identified structural homologs of the nucleotide binding domain of DisA and hence predicted the existence and extensive role of c-di-AMP in archaea and eubacteria. ${ }^{109}$ Consistent with this, c-di-AMP signaling has been reported in several Gram-positive bacteria and mycobacteria including Staphylococcus aureus, L. monocytogenes, Mycobacterium tuberculosis, Streptococcus pyogenes and Streptococcus pneumoniae and in some Gram-negative bacteria such as Chlamydia trachomatis. ${ }^{111-115}$ Fluctuations in the intracellular concentration of c-di-AMP has been implicated in various physiological roles including fatty acid synthesis, potassium ion transport, cell wall homeostasis, host type I interferon response induction, and biofilm formation. ${ }^{112,116-119}$

\section{c-di-AMP metabolism enzymes}

Very little is known about the environmental cues that elicit the production and degradation of c-di-AMP. Also the direct relationship between cellular c-di-AMP levels and the phenotypes observed remains to be elucidated. For example, low c-di-AMP levels sensitize $B$. subtilis and $L$. monocytogenes to cell wall targeting antibiotics. ${ }^{118,122}$ In $S$. aureus, high cellular c-di-AMP levels result in increased peptidoglycan crosslinking and resistance to cell wall targeting antibiotics. ${ }^{111}$ High c-di-AMP levels have also been shown to trigger stringent response resulting in an increase in the levels of the alarmone (p)ppGpp in S. aureus. ${ }^{18}$ Exogenously added c-di-AMP enhanced B. subtilis sporulation ${ }^{120}$ while overexpression of MsDisA in M. smegmatis (implying increased c-di-AMP levels) resulted in minute colonies ${ }^{121}$ (Fig. 12). Consequently, the cellular concentration of c-di-AMP is tightly regulated.

c-di-AMP is synthesized at the dimer interface of DisA_N (Pfam PF02457) domain-containing enzymes known as diadenylate cyclases (DAC) ${ }^{26}$ (Fig. 13 and 15). The active pocket contains two metal ions, which coordinate the phosphate moieties of two ATP molecules. A condensation of the two ATP molecules by active site residues forms c-di-AMP (Fig. 13). ${ }^{109,123}$ The DAC domain is conserved among the bacteria that utilize c-di-AMP signaling and it is characterized by a catalytic site with the conserved RHR (Arg-His-Arg) and DGA (Asp-Gly-Ala) motifs. ${ }^{109,123}$ Such enzymes have been reported in numerous bacteria including $B$. subtilis (DisA, ${ }^{109} \mathrm{CdaA}$ and $\mathrm{CdaS}^{124}$ ), S. pyogenes (CdaA), ${ }^{125}$ S. pneumoniae (CdaA) ${ }^{126}$ M. tuberculosis (MtDisA), ${ }^{113}$ C. trachomatis (CdaA) ${ }^{115}$ and L. monocytogenes (CdaA; PDB 4RV7). ${ }^{127}$ DAC enzymes 

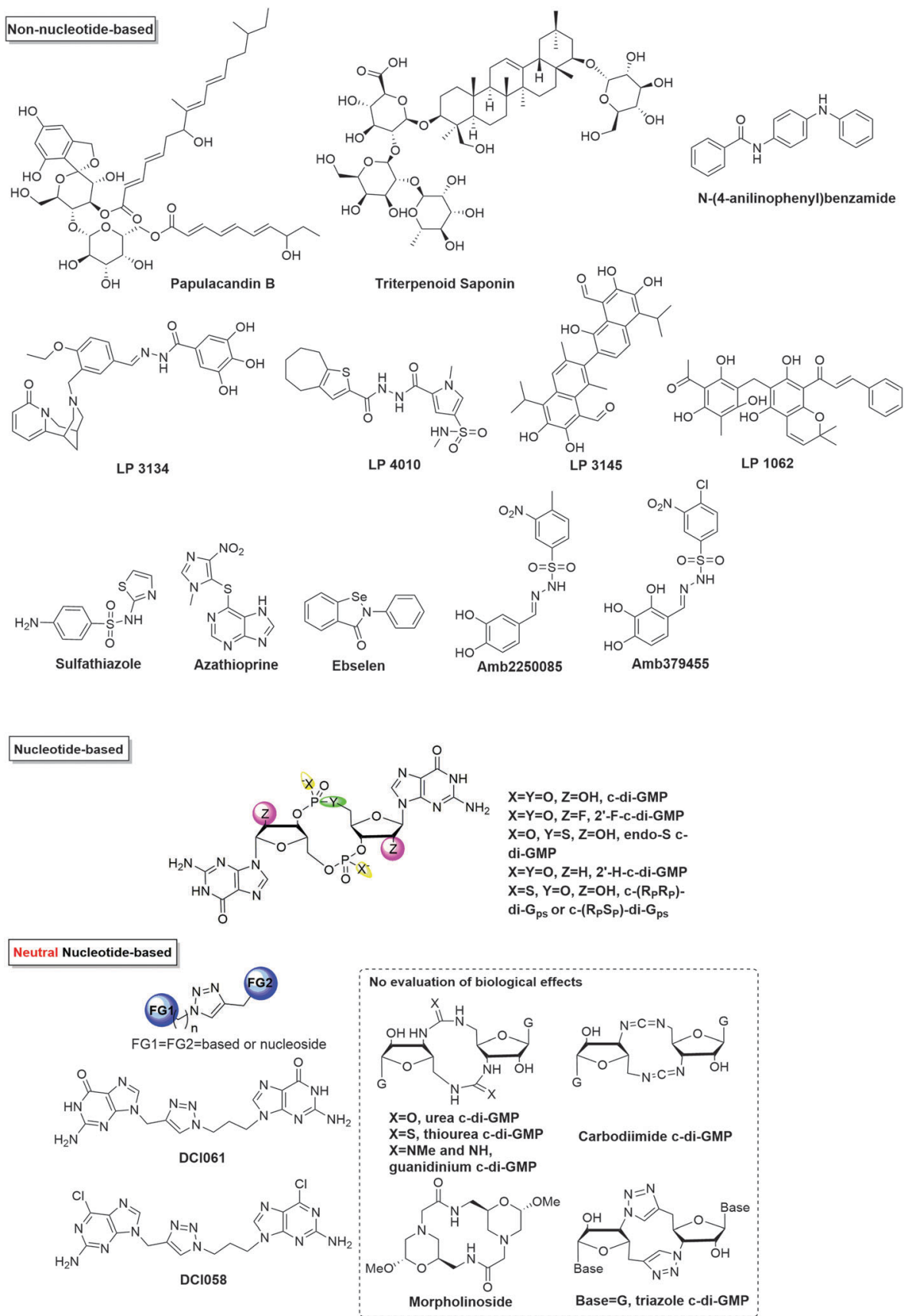

Fig. 10 Structures of small molecule inhibitors of c-di-GMP.

increase the local and/or global pools of c-di-AMP in bacteria. Attempts to delete the DAC gene from bacteria such as the human pathogen L. monocytogenes have been unsuccessful; ${ }^{112}$ pointing to the essentiality of c-di-AMP signaling in bacterial growth and physiology. Consequently, DAC has been viewed as a potential target for antibiotic development.

After effecting a signaling process or at higher than normal cellular concentrations, c-di-AMP is degraded by phosphodiesterase (PDE) to the linear pApA, ${ }^{17}$ a process which requires two metal ions ${ }^{129}$ (Fig. 14). Three families of phosphodiesterases have been identified to degrade c-di-AMP. Liang and co-workers identified B. subtilis GdpP (GGDEF domain protein-containing phosphodiesterase), belonging to the DHH-DHHA1 family of PDEs, as the first c-di-AMP PDE. ${ }^{129}$ The domain architecture of GdpP and its homologs are characterized by two transmembrane helical domains, the PAS (Per-Arnt-Sim) domain, a degenerate GGDEF domain and the DHH-DHHA1 catalytic domains. This family of PDEs has been shown in other bacteria such as $S$. aureus $(\mathrm{GdpP}){ }^{111}$ and $L$. monocytogenes (PdeA). ${ }^{118}$ A related family of PDEs is those that contain only the catalytic DHH-DHHA1 domain as found in B. burgdorferi (DhhP), ${ }^{130}$ S. pneumoniae (Pde1 and Pde2 $)^{126}$ and $M$. tuberculosis (CnpB). ${ }^{131,132}$ A third family of 

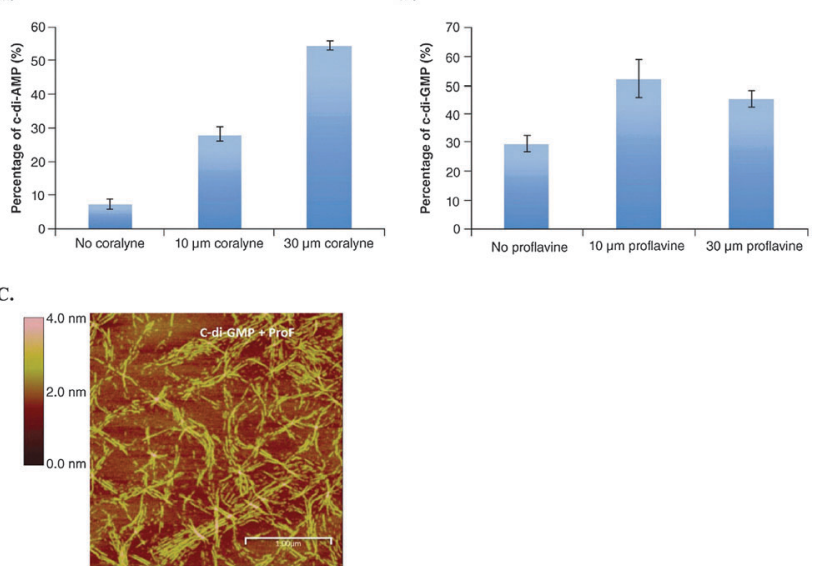

Fig. 11 (A) YybT cleavage of ${ }^{32} \mathrm{P}$-c-di-AMP in the presence and absence of coralyne. (B) YybT cleavage of ${ }^{32} \mathrm{P}-\mathrm{c}$-di-GMP in the presence and absence of proflavine. (C) Atomic force microscopy image of c-di-GMP proflavine aggregation. (A)-(C) Reproduced with permission from ref. 72 Copyright (C) 2016 Future Science.

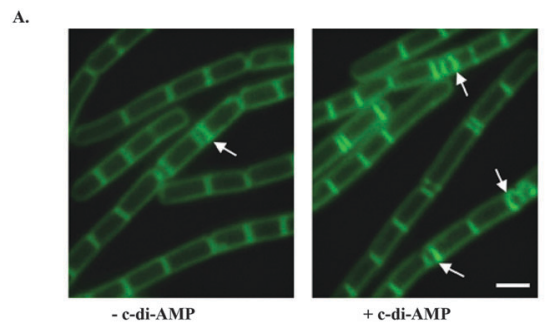

B.

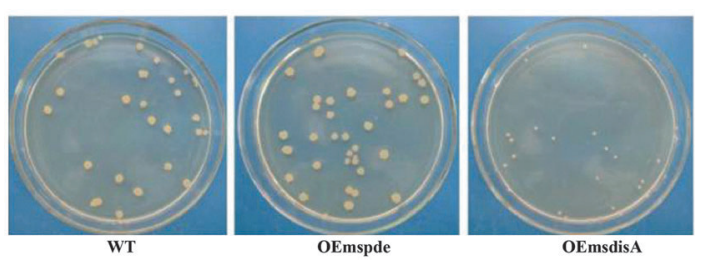

Fig. 12 (A) Addition of exogenous c-di-AMP (+ c-di-AMP) to B. subtilis cells showed an increase in sporulation characterized by the higher number of polar septa (white arrows) observed. (B) Overexpression of the DAC, DisA in M. smegmatis results in minute colonies compared to WT and PDE overexpression strains. (A) Reproduced with permission from ref. 120 (C) (2011) John Wiley \& Sons. (B) Reproduced with permission from ref. 121.

PDEs which belongs to the 7TMR-HD family was identified by Woodward and coworkers. ${ }^{133}$ The PDE, PgpH which was identified in L. monocytogenes, possesses a catalytic histidineaspartate (HD) domain. ${ }^{133}$ For a comprehensive overview of c-di-AMP PDEs, the reader is referred to a recent review by Huynh and Woodward. ${ }^{17}$ Another approach to decreasing intracellular c-di-AMP concentration could be seen in intracellular bacteria such as L. monocytogenes, C. trachomatis and M. tuberculosis. ${ }^{112,115,132}$ During infection, these pathogens secrete c-di-AMP into the host cytosol which induces type I IFN response (Fig. 15). Overall, DACs, PDEs and export proteins work together to maintain c-di-AMP homeostasis (Fig. 15).

\section{c-di-AMP receptors}

Binding of second messengers is a crucial step in signal transduction. After the discovery of c-di-AMP, a significant amount of effort was directed towards the identification of macromolecules that respond to fluctuations in cellular c-di-AMP concentration by binding to the second messenger. Several c-di-AMP binding partners have been identified encompassing proteins and RNA riboswitches (Fig. 15). Zhang et al. discovered the first c-di-AMP binding protein DarR, a TetR family transcription factor in M. smegmatis. ${ }^{116}$ The authors noted that the DNA binding activity of DarR was enhanced after binding c-di-AMP which results in the repression of expression of various genes, including genes associated with metabolism and transportation of fatty acids. ${ }^{116}$ The c-di-AMP binding proteins KtrA in $S$. aureus $^{136}$ and CabP in S. pneumoniae ${ }^{117}$ enable bacterial survival under low-potassium conditions and elevated c-di-AMP levels have been shown to inhibit potassium uptake. KdpD and CpaA which are $S$. aureus proteins predicted to be involved in potassium homeostasis and cation-proton antiporting respectively, have c-di-AMP binding sites. ${ }^{136}$ Also, the PII-like signal transduction proteins B. subtilis DarA ${ }^{137}$ and S. aureus PstA ${ }^{138}$ have been demonstrated to possess c-di-AMP binding affinity. L. monocytogenes pyruvate carboxylase (LmPC), which is essential for the growth of the human pathogen, was also shown to bind c-di-AMP. Binding of c-di-AMP was observed to inhibit the activity of LmPC. ${ }^{139}$ The $y d a O$ riboswitch class found in several bacteria has also been shown to have strong affinity for c-di-AMP ${ }^{140}$ compared to ATP which was earlier shown to be a ligand for the riboswitch. ${ }^{141}$

\section{Inhibitors of c-di-AMP}

c-di-AMP is undoubtedly an important second messenger in Gram-positive bacteria and the essentiality of c-di-AMP synthases has spurred the development of DAC inhibitors. c-di-AMP detection assays could facilitate inhibitor discovery. Bai and colleagues developed an ELISA-based assay for the detection and quantification of c-di-AMP using the c-di-AMP binding protein, CabP from S. pneumoniae. ${ }^{142}$ The assay was used to identify some c-di-AMP binding proteins. ${ }^{142}$ The Sintim group has also developed a surprisingly simple assay that uses commercially available coralyne to detect c-di-AMP and the group has used this assay to identify various inhibitors of DisA (Fig. 16). ${ }^{143}$

Cyclic di-AMP as stated earlier regulates cell wall homeostasis. With a majority of antibiotics targeting the bacterial cell wall, it is possible that inhibitors of c-di-AMP signaling could potentiate the activities of traditional cell wall-targeting antibiotics. However, there is a paucity of inhibitors against c-di-AMP metabolism enzymes $^{129,144}$ (Fig. 17 and Table 3). Sintim and co-workers established a high-throughput fluorescence assay for c-di-AMP detection using the coralyne fluorophore. ${ }^{143}$ After screening several compound libraries, three inhibitors of B. subtilis DisA were identified. ${ }^{144-146}$ Bromophenol-TH was the first documented DAC inhibitor. It was shown to specifically inhibit DisA with an $\mathrm{IC}_{50}$ of $56 \mu \mathrm{M} .{ }^{117}$ Suramin, an antiparasitic drug, and the tea polyphenol theaflavin digallate were also identified as potent DisA 


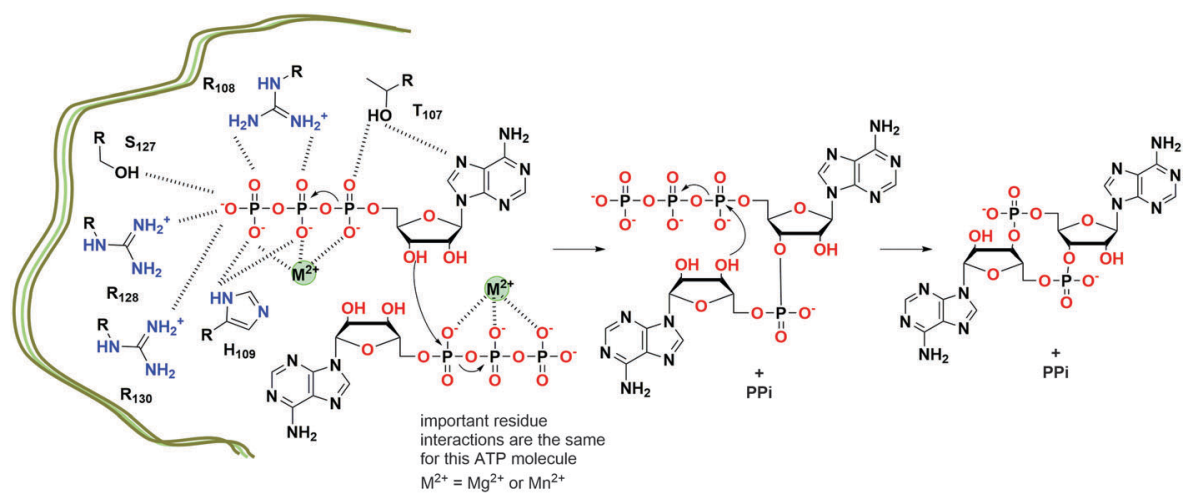

Fig. 13 Proposed mechanism of c-di-AMP formation by T. maritima DisA. ${ }^{128}$ (based on PDB 4YVZ and 4YXJ).
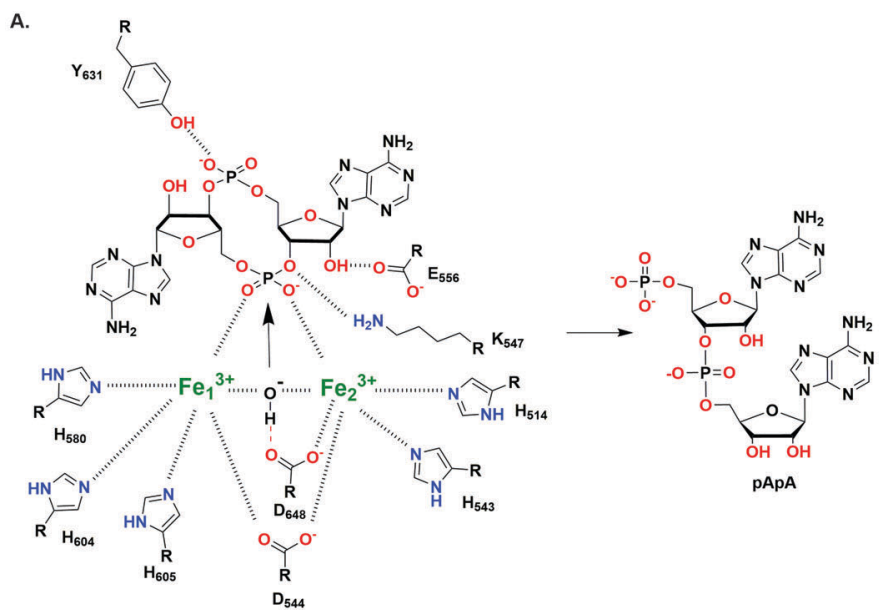

B.

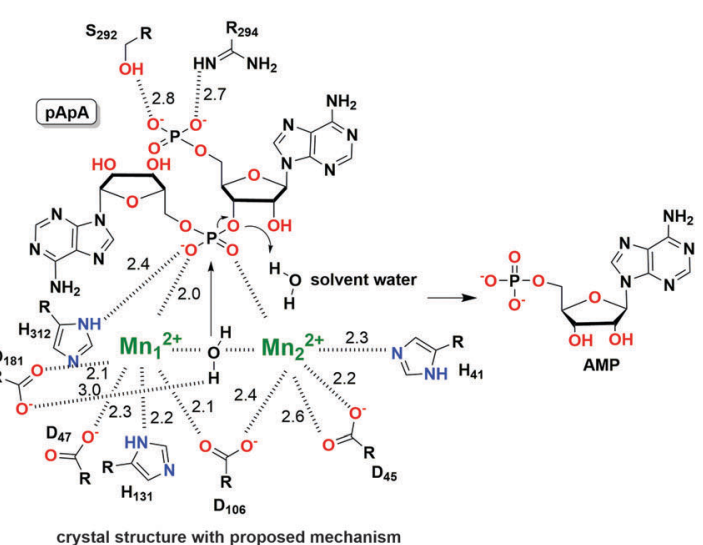

Fig. 14 (A) The hydrolysis mechanism of c-di-AMP by the HD domain phosphodiesterase from Listeria monocytogenes, PgpH. ${ }^{133}$ (PDB 4S1B). (B) The proposed hydrolysis mechanism of pApA by DHH-DHHA1 domain phosphodiesterase from Mycobacterium tuberculosis (Rv2837c). ${ }^{134}$ (PDB 5 CEU). (A) Adapted with permission from ref. 133 Copyright (C) 2015, National Academy of Sciences, USA (B) Adapted with permission from ref. 134. Copyright (C) 2016, The American Society for Biochemistry and Molecular Biology.

inhibitors with $\mathrm{IC}_{50}$ of $1.1 \mu \mathrm{M}$ and $3.4 \mu \mathrm{M}^{145,146}$ respectively. Witte and co-workers also reported that 3 '-deoxyATP inhibited Thermotoga maritima DisA with a $\mathrm{IC}_{50}$ of $3.8 \mu \mathrm{M} .{ }^{123}$ Liang and co-workers have shown that the stringent stress alarmone ppGpp could competitively inhibit B. subtilis GdpP (formerly YybT), with a $K_{i}$ of $35.9 \pm 7.2 \mu \mathrm{M} .{ }^{129}$ Additionally, ppGpp also inhibits GdpP in $S$. aureus with a $K_{i}$ of $129.7 \pm 42.8 \mu \mathrm{M}^{18}$ and $\mathrm{PgpH}$ in L. monocytogenes with a $\mathrm{IC}_{50}$ of $200-400 \mu \mathrm{M} .{ }^{133}$ Like c-di-GMP, the structure-activity relationship of c-di-AMP was also thoroughly studied by Strobel $e t$ al. and some of those analogs have been identified to be resistant to GdpP cleavage. ${ }^{147}$

\section{$3^{\prime} 3^{\prime}$-CGAMP}

In 2012, Mekalanos and colleagues explored the contribution of the Vibrio seventh pandemic island-1 (VSP-1) to pathogenesis and they discovered a new type of cyclic dinucleotide with 


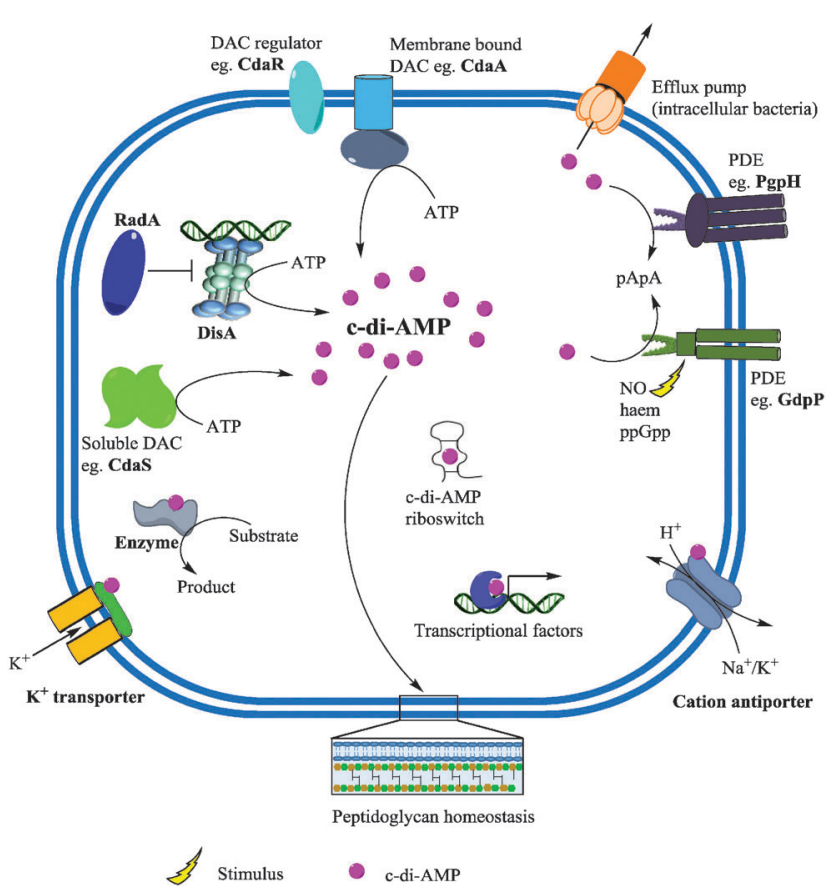

Fig. 15 c-di-AMP metabolism and effector functions. Membrane bound and/or soluble DACs synthesize c-di-AMP from ATP. c-di-AMP binds to receptor/target proteins and RNA to modulate physiological functions. Phosphodiesterases (PDE) degrade c-di-AMP into the linear PApA or AMP and the second messenger could also be secreted by the efflux pumps of some intracellular bacteria. Adapted from ref. 135 Copyright (C) 2015 Zheng, Ma, Wang, Xie, Ali and He.

hybrid bases, cyclic AMP-GMP (3'3'-cGAMP), in V. cholerae. $^{22}$ This new bacterial second messenger is synthesized by a novel class of dinucleotide cyclase $\mathrm{DncV},{ }^{22}$ which contains a conserved $\mathrm{G}[\mathrm{G} / \mathrm{S}] \mathrm{X}_{9-13} \mathrm{DX}[\mathrm{D} / \mathrm{E}]$ motif. DncV represses $V$. cholerae chemotaxis, a requirement for intestinal colonization. ${ }^{22}$ Subsequently, Jiang and colleagues reported three cGAMP-specific PDEs in $V$. cholerae with HD-GYP domains, named V-cGAP1/2/3. ${ }^{149}$ All of three PDEs were able to degrade $3^{\prime} 3^{\prime}$-cGAMP to the linear $5^{\prime}$-pApG but the $5^{\prime}$-nucleotidase activity of V-cGAP1 afforded it the capacity to cleave $5^{\prime}$-pApG to $5^{\prime}$-ApG in a second step. ${ }^{149}$ The protein receptors for $3^{\prime} 3^{\prime}$-cGAMP are still unknown. The first $3^{\prime} 3^{\prime}$-cGAMP riboswitch was developed based on the class I c-di-GMP riboswitch. In 2011, Strobel and colleagues found that a single C92U mutation in the ligand binding pocket enables the c-di-GMP class I riboswitch to bind to $3^{\prime} 3^{\prime}$-cGAMP. ${ }^{150}$ In 2015 , Hammond showed that Gram-negative bacterium Geobacter sulfurreducens produced $3^{\prime} 3^{\prime}$-cGAMP and that the GEMM-I (Genes for the Environment, Membranes, and Motility) class riboswitch was a $3^{\prime} 3^{\prime}$-cGAMP receptor that regulates electrophysiology genes. ${ }^{151}$ At the same time, Breaker and colleagues also reported the $3^{\prime} 3^{\prime}$-cGAMP riboswitch in Deltaproteobacteria that controls exoelectrogenesis. ${ }^{152}$

\section{2'3'-CGAMP}

In 2013, a cyclic dinucleotide second messenger, 2' $3^{\prime}$-cGAMP present mammals was uncovered by Ablasser, Hornung and colleagues. $^{23}$ One of the two phosphodiester bonds has a special linkage between $2^{\prime}-\mathrm{OH}$ of GMP and $5^{\prime}$-phosphate of AMP, distinguishing the mammalian $2^{\prime} 3^{\prime}$-cGAMP from other bacterial cyclic dinucleotides. ${ }^{153} 2^{\prime} 3^{\prime}$-cGAMP is synthesized from GTP and ATP by a cytoplasmic nucleotidyl transferase known as cyclic GMP-AMP synthase (cGAS). ${ }^{23}$ The $2^{\prime} 3^{\prime}$-cGAMP synthesis was postulated to occur in two steps. In a first step, GTP and ATP form the linear intermediate $\operatorname{pppGp}\left(2^{\prime}-5^{\prime}\right) \mathrm{A}$

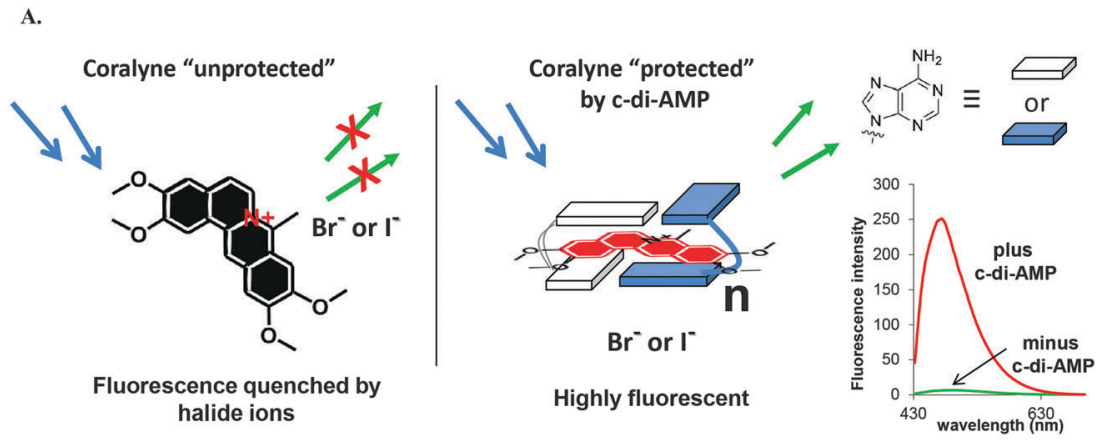

B.

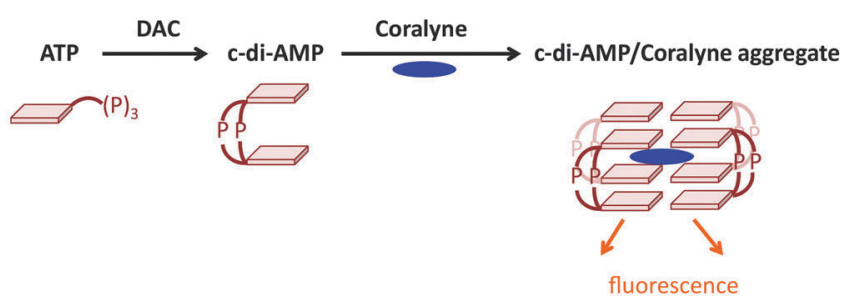

Fig. 16 (A) The principle of the coralyne fluorescence assay for C-di-AMP detection. (B) Schematic illustration of a DAC reaction showing the synthesis of C-di-AMP and the fluorescent c-di-AMP/coralyne complex. (A) Reproduced with permission from ref. 143 Copyright (C) 2014, American Chemical Society. (B) Reproduced with permission from ref. 144 Copyright (C) 2014, The Royal Society of Chemistry. 


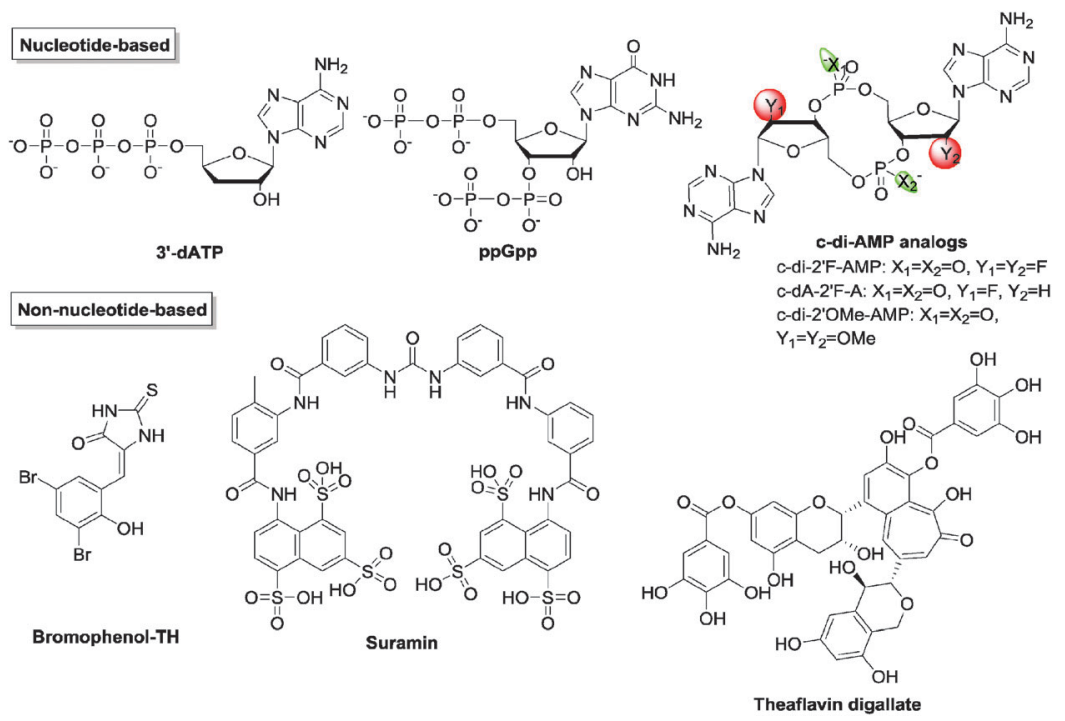

Fig. 17 Structures of representative c-di-AMP inhibitors.

Table 3 Summary of small molecules identified as c-di-AMP inhibitors

\begin{tabular}{|c|c|c|c|}
\hline Inhibitors & Functions & $\mathrm{IC}_{50}(\mu \mathrm{M})$ & Ref. \\
\hline \multicolumn{4}{|c|}{ Nucleotide-based inhibitors } \\
\hline $3^{\prime}$-dATP & Inhibits DAC (DisA) & 3 & 123 \\
\hline \multirow[t]{3}{*}{ ppGpp } & Inhibits PDE (YybT) & $35.9 \pm 7.2$ & 129 \\
\hline & Inhibits PDE (GdpP) & $129.7 \pm 42.8$ & 18 \\
\hline & Inhibits PDE (PgpH) & $200-400$ & 133 \\
\hline c-di-2'F-AMP & Inhibits PDE (GdpP) & $26 \pm 3.6$ & 147 \\
\hline $\mathrm{c}-\mathrm{dA}-2^{\prime} \mathrm{F}-\mathrm{A}$ & Inhibits PDE (GdpP) & $9.4 \pm 0.8$ & 147 \\
\hline c-di-2'OMe-AMP & Inhibits PDE (GdpP) & $15 \pm 2.5$ & 147 \\
\hline \multicolumn{4}{|c|}{ Non-nucleotide-based inhibitors } \\
\hline Bromophenol-TH & Inhibits DAC (DisA) & 56 & 148 \\
\hline Suramin & Inhibits DAC (DisA) & 1.1 & 145 \\
\hline Theaflavin digallate & Inhibits DAC (DisA) & 3.4 & 146 \\
\hline
\end{tabular}

which is cyclized to $2^{\prime} 3^{\prime}$-cGAMP in the second step. ${ }^{154}$ The enzymatic activity of cGAS is stimulated by double stranded DNA in the cytoplasm. When cGAS binds to cytoplasmic DNA, it synthesizes the signaling molecule $2^{\prime} 3^{\prime}$-cGAMP which induces immune response. ${ }^{155,156}$ In 2014, Mitchison and colleagues identified a glycoprotein on the plasma membrane and endoplasmic reticulum, ENPP1 that degraded $2^{\prime} 3^{\prime}$-cGAMP into AMP and GMP $\left(k_{\text {cat }}=12 \mathrm{~s}^{-1}, K_{\mathrm{m}}=20 \mu \mathrm{M}\right) .{ }^{157}$ ENPP1 was originally reported to be an ATP hydrolase $\left(k_{\text {cat }}=16 \mathrm{~s}^{-1}, K_{\mathrm{m}}=46 \mu \mathrm{M}\right)$. The crystal structure of ENPP1 (PDB 4HTW) revealed a $\mathrm{Ca}^{2+}$-binding domain and a site for two $\mathrm{Zn}^{2+}$ ions in its active pocket. ${ }^{158}$

\section{Type I interferon response}

One of the efficient methods for the host innate immune system to detect intracellular pathogens is to sense cytoplasmic DNA. As described previously, upon binding to cytoplasmic double stranded DNA, cGAS synthesizes the signaling molecule $2^{\prime} 3^{\prime}$ cGAMP. ${ }^{159} 2^{\prime} 3^{\prime}$-cGAMP binds to and activates STING (stimulator of interferon genes, also known as MITA, MPYS and ERIS), which is a 5 transmembrane domain protein that predominantly resides in the endoplasmic reticulum. ${ }^{23}$ Activated STING triggers the phosphorylation of transcriptional factor IFN regulatory factor (IRF3) by the kinase TANK-Binding Kinase 1 (TBK1). ${ }^{160,161}$ Phosphorylated IRF3 then translocates into the nucleus to induce the transcription of type I interferon genes (Fig. 18). The affinity of $2^{\prime} 3^{\prime}$-cGAMP for human STING has been shown to be very high, with a dissociation constant of $4.59 \mathrm{nM} .^{153}$

STING is able to sense cytoplasmic DNA directly, ${ }^{162}$ as well as the bacterial cyclic dinucleotides. ${ }^{163}$ Vance and colleagues showed the direct binding of STING to c-di-GMP and they obtained a $K_{\mathrm{d}}$ of $5 \mu \mathrm{M}$ by equilibrium dialysis. ${ }^{163} \mathrm{c}$-di-AMP is capable of inducing type I interferon response in a STINGdependent manner but with a lower binding affinity. ${ }^{115}$ Chen and colleagues measured the binding affinity of STING to 3'3-cGAMP and synthetic analogs $3^{\prime} 2^{\prime}$-cGAMP and $2^{\prime} 2^{\prime}$-cGAMP by ITC. $K_{\mathrm{d}}$ was measured as $1.04 \mu \mathrm{M}, 1.61 \mu \mathrm{M}$ and $287 \mathrm{nM}$. Their binding affinities are much lower than the host derived $2^{\prime} 3^{\prime}$-cGAMP. ${ }^{153}$

Small molecules that inhibit bacterial or human cyclic dinucleotide PDEs to increase cytosolic c-di-NMP in immune cells or activate cGAS to produce $2^{\prime} 3^{\prime}$-cGAMP or bind directly to STING to activate immune response could have anti-virulence properties. These molecules could however lead to unwanted prolonged inflammation and its associated unwanted effects such as pain, cancer and so on. On the other hand, it is also possible to develop c-di-NMP analogs that would bind to STING but prevent STING-related interferon response. Such molecules could have anti-inflammatory properties.

\section{Cyclic dinucleotides as anticancer agents or cancer immune adjuvants}

c-di-GMP has been shown to possess broad activities, such as inhibiting bacterial infection, ${ }^{164}$ stimulating host immune response, ${ }^{165}$ acting as an adjuvant for vaccination ${ }^{165}$ and inhibiting cancer cell proliferation. ${ }^{166}$ The potential of c-di-GMP as an agent against metastatic breast cancer, using a mice tumor model, has recently been demonstrated by Gravekamp. ${ }^{167}$ In this study, 


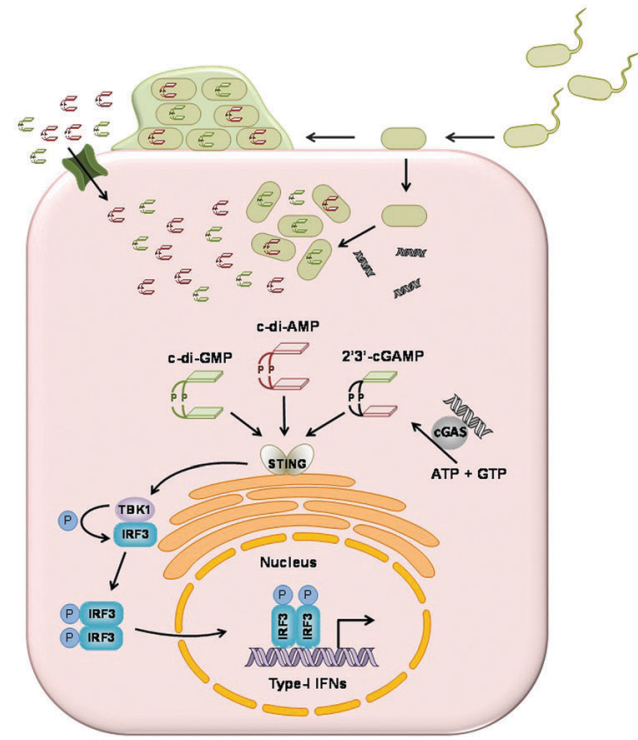

Fig. 18 Cyclic dinucleotides induce type I interferon response. cGAS detects cytoplasmic DNA and synthesizes 2'3'-cGAMP. 2'3'-cGAMP and c-di-AMP and c-di-GMP from bacteria bind to STING. Activated STING mediates the phosphorylation of IRF3 by TBK1. Phosphorylated IRF3 induces the transcription of type I interferon genes in the nucleus.

Gravekamp and co-workers firstly infected mice with metastatic breast cancer and then immunized them with an attenuated L. monocytogenes (LM)-based vaccine. After 19 days of treatment with low doses of c-di-GMP, the mice were able to survive with almost no metastases observed as well as improved T-cell responses. When treating mice infected with metastatic breast cancer by using one high dose and subsequent low doses of c-di-GMP, without the LM-based vaccine, they could achieve equal effectiveness against metastases. ${ }^{167}$ The real mechanism has not been fully elucidated. One possibility is that the LM-based vaccine infects myeloid-derived suppressor cells (MDSC) and c-di-GMP activates $\mathrm{T}$ cells, both toward reducing immune suppression and improving therapy. They also proposed that in the absence of the LM-based vaccine, high-dose of c-di-GMP would induce tumor cell death, while low-dose of c-di-GMP would induce activation of T-cells. ${ }^{167}$

Karaolis et al., in 2007, proposed that cyclic dinucleotides like c-di-GMP could be used as immunotherapeutic candidates in both humans and animals. ${ }^{165}$ However, the two phosphate groups on c-di-GMP inevitably prevents it from being developed as a drug candidate since it can barely cross cell membranes and may also be easily hydrolyzed. ${ }^{168}$ To address such issues, several groups have shown how to generate c-di-GMP inside host cells or transport c-di-GMP into mammalian cells for immunotherapy. ${ }^{168-172}$ Waters and co-workers used the adenovirus serotype 5 (Ad5) vector to transduce $d g c$ into mammalian cells, and thus increase intracellular c-di-GMP. ${ }^{169}$ In a more recent study, they combined VCA0848 (more potent $d g c$ ) with nonreplicating adenovirus serotype 5 (AdVCA0848) for an improved stimulation of innate immunity in mice. ${ }^{170}$ On the other hand, the Gursel group used the arginine peptide (positively charged) to complex with c-di-GMP (negatively charged) for delivery into mouse cells with enhanced immunostimulation of c-di-GMP being observed. ${ }^{168}$ In addition, Hayakawa loaded c-di-GMP on liposomes (YSK05 lipid), facilitating the delivery of c-di-GMP directly into the cytosol. As a result, c-di-GMP enhanced anticancer activity against malignant melanomas. ${ }^{171,172}$

Recently and unsurprisingly $2^{\prime} 3^{\prime}$-cGAMP has been identified as an immune adjuvant in mouse studies. ${ }^{173}$ Replacement of phosphodiester linkage by the phosphothioate bond resulted in a $2^{\prime} 3^{\prime}$-cGAMP analog resistant to hydrolysis while capable of activating human STING. ${ }^{157}$ Though such a $2^{\prime} 3^{\prime}$-cGAMP analog still possesses negative charges, it opens up the field to develop hSTING agonists as cancer immunotherapeutic drugs.

\section{Conclusions}

The importance of the physiological roles played by cyclic dinucleotides is well established. As a result significant effort has been directed towards identifying receptor/target proteins and RNA. The use of chemical probes represents one obvious approach to studying the molecular mechanisms underlying the phenotypes observed as a result of cyclic dinucleotide signaling. However, thus far only a handful of inhibitors have been uncovered for c-di-GMP and c-di-AMP metabolism. Furthermore, there are no inhibitors documented for the hybrid cyclic dinucleotide cGAMP $\left(2^{\prime} 3^{\prime}\right.$ and $\left.3^{\prime} 3^{\prime}\right)$ signaling. We believe that c-di-NMP signaling is on par with cNMP signaling or protein kinase signaling. Considering that inhibitors of these two signalings have had a tremendous impact on modern medicine, it is safe to predict that inhibitors of c-di-NMP signaling would also positively impact clinical practice. We hope that this review provides a rallying cry to other medicinal chemists to join the cyclic dinucleotide revolution and develop inhibitors against the various signaling pathways mediated by these fascinating molecules.

\section{Acknowledgements}

We thank the NSF (CHE grants 0746446 and 1307218) for funding our cyclic dinucleotide projects.

\section{Notes and references}

1 D. Kalia, G. Merey, S. Nakayama, Y. Zheng, J. Zhou, Y. Luo, M. Guo, B. T. Roembke and H. O. Sintim, Chem. Soc. Rev., 2013, 42, 305-341. 2 M. Gomelsky, Mol. Microbiol., 2011, 79, 562-565.

3 K. A. Lucas, G. M. Pitari, S. Kazerounian, I. Ruiz-Stewart, J. Park, S. Schulz, K. P. Chepenik and S. A. Waldman, Pharmacol. Rev., 2000, 52, 375-414.

4 J. N. Marden, Q. Dong, S. Roychowdhury, J. E. Berleman and C. E. Bauer, Mol. Microbiol., 2011, 79, 600-615.

5 C. Pesavento and R. Hengge, Curr. Opin. Microbiol., 2009, 12, 170-176.

6 V. Hauryliuk, G. C. Atkinson, K. S. Murakami, T. Tenson and K. Gerdes, Nat. Rev. Microbiol., 2015, 13, 298-309.

7 I. M. Ernst, R. Fliegert and A. H. Guse, Front. Immunol., 2013 , 4, 259.

8 X. Gu, Z. Yang, L. Zhang, S. Kunerth, R. Fliegert, K. Weber and A. H. Guse, J. Med. Chem., 2004, 47, 5674-5682.

9 P. C. Lee, B. R. Bochner and B. N. Ames, J. Biol. Chem., 1983, 258, 6827-6834. 
10 A. Varshavsky, Cell, 1983, 34, 711-712.

11 L. L. Kisselev, J. Justesen, A. D. Wolfson and L. Y. Frolova, FEBS Lett., 1998, 427, 157-163.

12 R. Hengge, A. Gründling, U. Jenal, R. Ryan and F. Yildiz, J. Bacteriol., 2016, 198, 15-26.

13 U. Römling, M. Y. Galperin and M. Gomelsky, Microbiol. Mol. Biol. Rev., 2013, 77, 1-52.

14 T. H. Pham, Z. X. Liang, E. Marcellin and M. S. Turner, Curr. Genet., 2016, 1-8.

15 Z. X. Liang, Nat. Prod. Rep., 2015, 32, 663-683.

16 D. G. Ha and G. A. O'Toole, Microbiol. Spectrum, 2015, 3, MB-00032014.

17 T. N. Huynh and J. J. Woodward, Curr. Opin. Microbiol., 2016, 30, 22-29.

18 R. M. Corrigan, L. Bowman, A. R. Willis, V. Kaever and A. Gründling, J. Biol. Chem., 2015, 290, 5826-5839.

19 D. Gao, T. Li, X. D. Li, X. Chen, Q. Z. Li, M. Wight-Carter and Z. J. Chen, Proc. Natl. Acad. Sci. U. S. A., 2015, 112, E5699-E5705.

20 P. Ross, H. Weinhouse, Y. Aloni, D. Michaeli, P. Weinbergerohana, R. Mayer, S. Braun, E. Devroom, G. A. Vandermarel, J. H. Vanboom and M. Benziman, Nature, 1987, 325, 279-281.

21 U. Römling, Sci. Signaling, 2008, 1, pe39.

22 B. W. Davies, R. W. Bogard, T. S. Young and J. J. Mekalanos, Cell, 2012, 149, 358-370.

23 A. Ablasser, M. Goldeck, T. Cavlar, T. Deimling, G. Witte, I. Roehl, K.-P. Hopfner, J. Ludwig and V. Hornung, Nature, 2013, 498, 380-384.

24 X. Cai, Y. H. Chiu and Z. J. Chen, Mol. Cell, 2014, 54, 289-296.

25 T. S. Xiao and K. A. Fitzgerald, Mol. Cell, 2013, 51, 135-139.

26 R. M. Corrigan and A. Gründling, Nat. Rev. Microbiol., 2013, 11, 513-524.

27 G. B. Hecht and A. Newton, J. Bacteriol., 1995, 177, 6223-6229.

28 R. Tal, H. C. Wong, R. Calhoon, D. Gelfand, A. L. Fear, G. Volman, R. Mayer, P. Ross, D. Amikam, H. Weinhouse, A. Cohen, S. Sapir, P. Ohana and M. Benziman, J. Bacteriol., 1998, 180, 4416-4425.

29 N. De, M. V. A. S. Navarro, R. V. Raghavan and H. Sondermann, J. Mol. Biol., 2009, 393, 619-633.

30 R. Simm, M. Morr, A. Kader, M. Nimtz and U. Römling, Mol. Microbiol., 2004, 53, 1123-1134.

31 M. Tarnawski, T. R. Barends and I. Schlichting, Acta Crystallogr., Sect. D: Biol. Crystallogr., 2015, 71, 2158-2177.

32 J. L. Hunter, G. B. Severin, B. J. Koestler and C. M. Waters, $B M C$ Microbiol., 2014, 14, 22.

33 D. Pérez-Mendoza, S. J. Coulthurst, S. Humphris, E. Campbell, M. Welch, I. K. Toth and G. P. Salmond, Mol. Microbiol., 2011, 82, 719-733.

34 N. De, M. Pirruccello, P. V. Krasteva, N. Bae, R. V. Raghavan and H. Sondermann, PLoS Biol., 2008, 6, 601-617.

35 C. Chan, R. Paul, D. Samoray, N. C. Amiot, B. Giese, U. Jenal and T. Schirmer, Proc. Natl. Acad. Sci. U. S. A., 2004, 101, 17084-17089.

36 M. W. Chen, M. Kotaka, C. Vonrhein, G. Bricogne, F. Rao, M. L. C. Chuah, D. Svergun, G. Schneider, Z.-X. Liang and J. Lescar, J. Bacteriol., 2012, 194, 4837-4846.

37 F. Rao, Y. Yang, Y. Qi and Z. X. Liang, J. Bacteriol., 2008, 190, $3622-3631$.

38 M. W. Orr, G. P. Donaldson, G. B. Severin, J. Wang, H. O. Sintim, C. M. Waters and V. T. Lee, Proc. Natl. Acad. Sci. U. S. A., 2015, 112, E5048-E5057.

39 D. Cohen, U. Mechold, H. Nevenzal, Y. Yarmiyhu, T. E. Randall, D. C. Bay, J. D. Rich, M. R. Parsek, V. Kaever, J. J. Harrison and E. Banin, Proc. Natl. Acad. Sci. U. S. A., 2015, 112, 11359-11364.

40 S. Ghosh and M. P. Deutscher, Proc. Natl. Acad. Sci. U. S. A., 1999, 96, 4372-4377.

41 S. K. Niyogi and A. K. Datta, J. Biol. Chem., 1975, 250, 7307-7312.

42 D. Yu and M. P. Deutscher, J. Bacteriol., 1995, 177, 4137-4139.

43 M. Y. Galperin, A. N. Nikolskaya and E. V. Koonin, FEMS Microbiol. Lett., 2001, 204, 213-214.

44 R. P. Ryan, Y. Fouhy, J. F. Lucey, L. C. Crossman, S. Spiro, Y. W. He, L. H. Zhang, S. Heeb, M. Camara, P. Williams and J. M. Dow, Proc. Natl. Acad. Sci. U. S. A., 2006, 103, 6712-6717.

45 A. L. Lovering, M. J. Capeness, C. Lambert, L. Hobley and R. E. Sockett, mBio, 2011, 2, e00163.

46 D. Bellini, D. L. Caly, Y. McCarthy, M. Bumann, S. Q. An, J. M. Dow, R. P. Ryan and M. A. Walsh, Mol. Microbiol., 2014, 91, 26-38.
47 S. Rinaldo, A. Paiardini, V. Stelitano, P. Brunotti, L. Cervoni, S. Fernicola, C. Protano, M. Vitali, F. Cutruzzolà and G. Giardina, J. Bacteriol., 2015, 197, 1525-1535.

48 C. D. Boyd and G. A. O’Toole, Annu. Rev. Cell Dev. Biol., 2012, 28, 439-462.

49 A. J. Wolfe and K. L. Visick, J. Bacteriol., 2008, 190, 463-475.

50 C. Lori, S. Ozaki, S. Steiner, R. Böhm, S. Abel, B. N. Dubey, T. Schirmer, S. Hiller and U. Jenal, Nature, 2015, 523, 236-239.

51 A. Duerig, S. Abel, M. Folcher, M. Nicollier, T. Schwede, N. Amiot, B. Giese and U. Jenal, Genes Dev., 2009, 23, 93-104.

52 R. Hengge, Nat. Rev. Microbiol., 2009, 7, 263-273.

53 Y. Kumagai, J. Matsuo, Y. Hayakawa and Y. Rikihisa, J. Bacteriol., 2010, 192, 4122-4133.

54 D. Amikam and M. Y. Galperin, Bioinformatics, 2006, 22, 3-6.

55 D. A. Ryjenkov, R. Simm, U. Römling and M. Gomelsky, J. Biol. Chem., 2006, 281, 30310-30314.

56 J. C. Whitney, G. B. Whitfield, L. S. Marmont, P. Yip, A. M. Neculai, Y. D. Lobsanov, H. Robinson, D. E. Ohman and P. L. Howell, J. Biol. Chem., 2015, 290, 12451-12462.

57 M. Christen, B. Christen, M. G. Allan, M. Folcher, P. Jenö, S. Grzesiek and U. Jenal, Proc. Natl. Acad. Sci. U. S. A., 2007, 104, 4112-4117.

58 J. T. Pratt, R. Tamayo, A. D. Tischler and A. Camilli, J. Biol. Chem., 2007, 282, 12860-12870.

59 C. Chan, R. Paul, D. Samoray, N. C. Amiot, B. Giese, U. Jenal and T. Schirmer, Proc. Natl. Acad. Sci. U. S. A., 2004, 101, 17084-17089.

60 M. V. A. S. Navarro, N. De, N. Bae, Q. Wang and H. Sondermann, Structure, 2009, 17, 1104-1116.

61 S. Ozaki, A. Schalch-Moser, L. Zumthor, P. Manfredi, A. Ebbensgaard, T. Schirmer and U. Jenal, Mol. Microbiol., 2014, 94, 580-594.

62 T. Su, S. Liu, K. Wang, K. Chi, D. Zhu, T. Wei, Y. Huang, L. Guo, W. Hu, S. Xu, Z. Lin and L. Gu, J. Struct. Biol., 2015, 192, 1-13.

63 B. Y. Matsuyama, P. V. Krasteva, C. Baraquet, C. S. Harwood, H. Sondermann and M. V. Navarro, Proc. Natl. Acad. Sci. U. S. A., 2016, 113, E209-E218.

64 P. V. Krasteva, J. C. Fong, N. J. Shikuma, S. Beyhan, M. V. Navarro, F. H. Yildiz and H. Sondermann, Science, 2010, 327, 866-868.

65 N. Sudarsan, E. R. Lee, Z. Weinberg, R. H. Moy, J. N. Kim, K. H. Link and R. R. Breaker, Science, 2008, 321, 411-413.

66 E. R. Lee, J. L. Baker, Z. Weinberg, N. Sudarsan and R. R. Breaker, Science, 2010, 329, 845-848.

67 Z. Zhang, B. L. Gaffney and R. A. Jones, J. Am. Chem. Soc., 2004, 126, 16700-16701.

68 J. Wang, J. Zhou, G. P. Donaldson, S. Nakayama, L. Yan, Y. F. Lam, V. T. Lee and H. O. Sintim, J. Am. Chem. Soc., 2011, 133, 9320-9330.

69 J. Zhou, D. A. Sayre, J. Wang, N. Pahadi and H. O. Sintim, Molecules, 2012, 17, 13376-13389.

70 J. Zhao, E. Veliath, S. Kim, B. L. Gaffney and R. A. Jones, Nucleosides, Nucleotides Nucleic Acids, 2009, 28, 352-378.

71 K. Sambanthamoorthy, R. E. Sloup, V. Parashar, J. M. Smith, E. E. Kim, M. F. Semmelhack, M. B. Neiditch and C. M. Waters, Antimicrob. Agents Chemother., 2012, 56, 5202-5211.

72 S. Nakayama, J. Zhou, Y. Zheng, H. Szmacinski and H. O. Sintim, Future Sci. OA, 2016, 2, DOI: 10.4155/fso.4115.4193.

73 S. Nakayama, Y. Luo, J. Zhou, T. K. Dayie and H. O. Sintim, Chem. Commun., 2012, 48, 9059-9061.

74 E. J. Diner, D. L. Burdette, S. C. Wilson, K. M. Monroe, C. A. Kellenberger, M. Hyodo, Y. Hayakawa, M. C. Hammond and R. E. Vance, Cell Rep., 2013, 3, 1355-1361.

75 C. A. Kellenberger, S. C. Wilson, J. Sales-Lee and M. C. Hammond, J. Am. Chem. Soc., 2013, 135, 4906-4909.

76 C. A. Kellenberger, C. Chen, A. T. Whiteley, D. A. Portnoy and M. C. Hammond, J. Am. Chem. Soc., 2015, 137, 6432-6435.

77 G. Tsuji and H. O. Sintim, Mol. BioSyst., 2016, 12, 773-777.

78 S. Nakayama, I. Kelsey, J. Wang, K. Roelofs, B. Stefane, Y. Luo, V. T. Lee and H. O. Sintim, J. Am. Chem. Soc., 2011, 133, 4856-4864.

79 S. Nakayama, I. Kelsey, J. Wang and H. O. Sintim, Chem. Commun., 2011, 47, 4766-4768.

80 B. T. Roembke, J. Zhou, Y. Zheng, D. Sayre, A. Lizardo, L. Bernard and H. O. Sintim, Mol. BioSyst., 2014, 10, 1568-1575.

81 S. Fernicola, A. Paiardini, G. Giardina, G. Rampioni, L. Leoni, F. Cutruzzolà and S. Rinaldo, J. Bacteriol., 2016, 198, 147-156.

82 O. J. Lieberman, M. W. Orr, Y. Wang and V. T. Lee, ACS Chem. Biol., 2014, 9, 183-192. 
83 M. Y. Galperin, A. N. Nikolskaya and E. V. Koonin, FEMS Microbiol. Lett., 2001, 203, 11-21.

84 A. Kader, R. Simm, U. Gerstel, M. Morr and U. Römling, Mol. Microbiol., 2006, 60, 602-616.

85 Y. Li, S. Heine, M. Entian, K. Sauer and N. Frankenberg-Dinkel, J. Bacteriol., 2013, 195, 3531-3542.

86 O. J. Lieberman, J. J. DeStefano and V. T. Lee, PLoS One, 2013, 8, e53689.

87 K. Sambanthamoorthy, R. E. Sloup, V. Parashar, J. M. Smith, E. E. Kim, M. F. Semmelhack, M. B. Neiditch and C. M. Waters, Antimicrob. Agents Chemother., 2012, 56, 5202-5211.

88 K. Sambanthamoorthy, C. Luo, N. Pattabiraman, X. Feng, B. Koestler, C. M. Waters and T. J. Palys, Biofouling, 2014, 30, 17-28.

89 D. Antoniani, E. Rossi, S. Rinaldo, P. Bocci, M. Lolicato, A. Paiardini, N. Raffaelli, F. Cutruzzolà and P. Landini, Appl. Microbiol. Biotechnol., 2013, 97, 7325-7336.

90 D. Antoniani, P. Bocci, A. Maciag, N. Raffaelli and P. Landini, Appl. Microbiol. Biotechnol., 2010, 85, 1095-1104.

91 C. A. Shanahan and S. A. Strobel, Org. Biomol. Chem., 2012, 10, 9113-9129.

92 J. Zhou, S. Watt, J. Wang, S. Nakayama, D. A. Sayre, Y. F. Lam, V. T. Lee and H. O. Sintim, Bioorg. Med. Chem., 2013, 21, 4396-4404.

93 P. Ohana, D. P. Delmer, R. W. Carlson, J. Glushka, P. Azadi, T. Bacic and M. Benziman, Plant Cell Physiol., 1998, 39, 144-152.

94 C. A. Shanahan, B. L. Gaffney, R. A. Jones and S. A. Strobel, Biochemistry, 2013, 52, 365-377.

95 J. Wang, J. Zhou, G. P. Donaldson, S. Nakayama, L. Yan, Y. F. Lam, V. T. Lee and H. O. Sintim, J. Am. Chem. Soc., 2011, 133, 9320-9330.

96 C. A. Shanahan, B. L. Gaffney, R. A. Jones and S. A. Strobel, J. Am. Chem. Soc., 2011, 133, 15578-15592.

97 K. D. Smith, S. V. Lipchock and S. A. Strobel, Biochemistry, 2012, 51, 425-432.

98 S. L. Kuchma, A. E. Ballok, J. H. Merritt, J. H. Hammond, W. Lu, J. D. Rabinowitz and G. A. O’Toole, J. Bacteriol., 2010, 192, 2950-2964.

99 J. W. Hickman, D. F. Tifrea and C. S. Harwood, Proc. Natl. Acad. Sci. U. S. A., 2005, 102, 14422-14427.

100 H. Kulesekara, V. Lee, A. Brencic, N. Liberati, J. Urbach, S. Miyata, D. G. Lee, A. N. Neely, M. Hyodo, Y. Hayakawa, F. M. Ausubel and S. Lory, Proc. Natl. Acad. Sci. U. S. A., 2006, 103, 2839-2844.

101 W. J. Sandborn, E. C. Van O, B. J. Zins, W. J. Tremaine, D. C. Mays and J. J. Lipsky, Gastroenterology, 1995, 109, 1808-1817.

102 G. K. Azad and R. S. Tomar, Mol. Biol. Rep., 2014, 41, 4865-4879.

103 S. Thangamani, W. Younis and M. N. Seleem, Sci. Rep., 2015, $5,11596$.

104 Y. Ishihara, M. Hyodo, Y. Hayakawa, T. Kamegaya, K. Yamada, A. Okamoto, T. Hasegawa and M. Ohta, FEMS Microbiol. Lett., 2009, 301, 193-200.

105 B. L. Gaffney and R. A. Jones, Org. Lett., 2014, 16, 158-161.

106 T. Fujino, K. Okada and H. Isobe, Tetrahedron Lett., 2014, 55, 2659-2661.

107 C. Kinzie, A. Steele, S. Pasciolla and W. Wuest, Tetrahedron Lett., 2014, 55, 4966-4968.

108 S. Fernicola, I. Torquati, A. Paiardini, G. Giardina, G. Rampioni, M. Messina, L. Leoni, F. Del Bello, R. Petrelli, S. Rinaldo, L. Cappellacci and F. Cutruzzolà, J. Med. Chem., 2015, 58, 8269-8284.

109 G. Witte, S. Hartung, K. Buettner and K.-P. Hopfner, Mol. Cell, 2008, 30, 167-178.

110 M. Bejerano-Sagie, Y. Oppenheimer-Shaanan, I. Berlatzky, A. Rouvinski, M. Meyerovich and S. Ben-Yehuda, Cell, 2006, 125, 679-690.

111 R. M. Corrigan, J. C. Abbott, H. Burhenne, V. Kaever and A. Gründling, PLoS Pathog., 2011, 7, e1002217.

112 J. J. Woodward, A. T. Iavarone and D. A. Portnoy, Science, 2010, 328, 1703-1705.

113 Y. Bai, J. Yang, X. Zhou, X. Ding, L. E. Eisele and G. Bai, PLoS One, 2012, 7, e35206.

114 T. Kamegaya, K. Kuroda and Y. Hayakawa, Nagoya J. Med. Sci., 2011, 73, 49-57.

115 J. R. Barker, B. J. Koestler, V. K. Carpenter, D. L. Burdette, C. M. Waters, R. E. Vance and R. H. Valdivia, mBio, 2013, 4, e00018.

116 L. Zhang, W. Li and Z. G. He, J. Biol. Chem., 2013, 288, 3085-3096.

117 Y. Bai, J. Yang, T. M. Zarrella, Y. Zhang, D. W. Metzger and G. Bai, J. Bacteriol., 2014, 196, 614-623.

118 C. E. Witte, A. T. Whiteley, T. P. Burke, J.-D. Sauer, D. A. Portnoy and J. J. Woodward, mBio, 2013, 4, e00282.
119 X. Peng, Y. Zhang, G. Bai, X. Zhou and H. Wu, Mol. Microbiol., 2016, 99, 945-959.

120 Y. Oppenheimer-Shaanan, E. Wexselblatt, J. Katzhendler, E. Yavin and S. Ben-Yehuda, EMBO Rep., 2011, 12, 594-601.

121 Q. Tang, Y. Luo, C. Zheng, K. Yin, M. K. Ali, X. Li and J. He, Int. J. Biol. Sci., 2015, 11, 813-824.

122 Y. Luo and J. D. Helmann, Mol. Microbiol., 2012, 83, 623-639.

123 M. Müller, T. Deimling, K. P. Hopfner and G. Witte, Biochem. J., 2015, 469, 367-374.

124 F. M. Mehne, K. Schröder-Tittmann, R. T. Eijlander, C. Herzberg, L. Hewitt, V. Kaever, R. J. Lewis, O. P. Kuipers, K. Tittmann and J. Stülke, J. Biol. Chem., 2014, 289, 21098-21107.

125 T. Kamegaya, K. Kuroda and Y. Hayakawa, Nagoya J. Med. Sci., 2011, 73, 49-57.

126 Y. Bai, J. Yang, L. E. Eisele, A. J. Underwood, B. J. Koestler, C. M. Waters, D. W. Metzger and G. Bai, J. Bacteriol., 2013, 195, 5123-5132.

127 J. Rosenberg, A. Dickmanns, P. Neumann, K. Gunka, J. Arens, V. Kaever, J. Stülke, R. Ficner and F. M. Commichau, J. Biol. Chem., 2015, 290, 6596-6606.

128 M. Müller, T. Deimling, K. P. Hopfner and G. Witte, Biochem. J., 2015, 469, 367-374.

129 F. Rao, R. Y. See, D. Zhang, D. C. Toh, Q. Ji and Z.-X. Liang, J. Biol. Chem., 2010, 285, 473-482.

130 M. Ye, J.-J. Zhang, X. Fang, G. B. Lawlis, B. Troxell, Y. Zhou, M. Gomelsky, Y. Lou and X. F. Yang, Infect. Immun., 2014, 82, 1840-1849.

131 K. Manikandan, V. Sabareesh, N. Singh, K. Saigal, U. Mechold and K. M. Sinha, PLoS One, 2014, 9, e86096.

132 J. Yang, Y. Bai, Y. Zhang, V. D. Gabrielle, L. Jin and G. Bai, Mol. Microbiol., 2014, 93, 65-79.

133 T. N. Huynh, S. Luo, D. Pensinger, J. D. Sauer, L. Tong and J. J. Woodward, Proc. Natl. Acad. Sci. U. S. A., 2015, 112, E747-E756.

134 Q. He, F. Wang, S. Liu, D. Zhu, H. Cong, F. Gao, B. Li, H. Wang, Z. Lin, J. Liao and L. Gu, J. Biol. Chem., 2016, 291, 3668-3681.

135 C. Zheng, Y. Ma, X. Wang, Y. Xie, M. K. Ali and J. He, Front. Microbiol., 2015, 6, 908.

136 R. M. Corrigan, I. Campeotto, T. Jeganathan, K. G. Roelofs, V. T. Lee and A. Gründling, Proc. Natl. Acad. Sci. U. S. A., 2013, 110, 9084-9089.

137 J. Gundlach, A. Dickmanns, K. Schröder-Tittmann, P. Neumann, J. Kaesler, J. Kampf, C. Herzberg, E. Hammer, F. Schwede, V. Kaever, K. Tittmann, J. Stülke and R. Ficner, J. Biol. Chem., 2015, 290, 3069-3080.

138 I. Campeotto, Y. Zhang, M. G. Mladenov, P. S. Freemont and A. Gründling, J. Biol. Chem., 2015, 290, 2888-2901.

139 K. Sureka, P. H. Choi, M. Precit, M. Delince, D. A. Pensinger, T. N. Huynh, A. R. Jurado, Y. A. Goo, M. Sadilek, A. T. Iavarone, J. D. Sauer, L. Tong and J. J. Woodward, Cell, 2014, 158, 1389-1401.

140 J. W. Nelson, N. Sudarsan, K. Furukawa, Z. Weinberg, J. X. Wang and R. R. Breaker, Nat. Chem. Biol., 2013, 9, 834-839.

141 P. Y. Watson and M. J. Fedor, Nat. Chem. Biol., 2012, 8, 963-965.

142 A. J. Underwood, Y. Zhang, D. W. Metzger and G. Bai, J. Microbiol. Methods, 2014, 107, 58-62.

143 J. Zhou, D. A. Sayre, Y. Zheng, H. Szmacinski and H. O. Sintim, Anal. Chem., 2014, 86, 2412-2420.

144 Y. Zheng, J. Zhou, D. A. Sayre and H. O. Sintim, Chem. Commun., 2014, 50, 11234-11237.

145 C. Opoku-Temeng and H. O. Sintim, Chem. Commun., 2016, 52, 3754-3757.

146 C. Opoku-Temeng and H. O. Sintim, Sci. Rep., 2016, 6, 25445, DOI: $10.1038 /$ srep25445.

147 R. E. Meehan, C. D. Torgerson, B. L. Gaffney, R. A. Jones and S. A. Strobel, Biochemistry, 2016, 55, 837-849.

148 Y. Zheng, J. Zhou, D. A. Sayre and H. O. Sintim, Chem. Commun., 2014, 50, 11234-11237.

149 J. Gao, J. Tao, W. Liang, M. Zhao, X. Du, S. Cui, H. Duan, B. Kan, X. Su and Z. Jiang, Cell Res., 2015, 25, 539-550.

150 C. A. Shanahan, B. L. Gaffney, R. A. Jones and S. A. Strobel, J. Am. Chem. Soc., 2011, 133, 15578-15592.

151 C. A. Kellenberger, S. C. Wilson, S. F. Hickey, T. L. Gonzalez, Y. Su, Z. F. Hallberg, T. F. Brewer, A. T. Iavarone, H. K. Carlson, Y. F. Hsieh and M. C. Hammond, Proc. Natl. Acad. Sci. U. S. A., 2015, 112, 5383-5388. 
152 J. W. Nelson, N. Sudarsan, G. E. Phillips, S. Stav, C. E. Lünse, P. J. McCown and R. R. Breaker, Proc. Natl. Acad. Sci. U. S. A., 2015, 112, 5389-5394.

153 X. Zhang, H. Shi, J. Wu, X. Zhang, L. Sun, C. Chen and Z. J. Chen, Mol. Cell, 2013, 51, 226-235.

154 P. Gao, M. Ascano, Y. Wu, W. Barchet, B. L. Gaffney, T. Zillinger, A. A. Serganov, Y. Liu, R. A. Jones, G. Hartmann, T. Tuschl and D. J. Patel, Cell, 2013, 153, 1094-1107.

155 J. Wu, L. Sun, X. Chen, F. Du, H. Shi, C. Chen and Z. J. Chen, Science, 2013, 339, 826-830.

156 L. Sun, J. Wu, F. Du, X. Chen and Z. J. Chen, Science, 2013, 339, 786-791.

157 L. Li, Q. Yin, P. Kuss, Z. Maliga, J. L. Millán, H. Wu and T. J. Mitchison, Nat. Chem. Biol., 2014, 10, 1043-1048.

158 K. Kato, H. Nishimasu, S. Okudaira, E. Mihara, R. Ishitani, J. Takagi, J. Aoki and O. Nureki, Proc. Natl. Acad. Sci. U. S. A., 2012, 109, 16876-16881.

159 X. Zhang, J. Wu, F. Du, H. Xu, L. Sun, Z. Chen, C. A. Brautigam and Z. J. Chen, Cell Rep., 2014, 6, 421-430.

160 V. Hornung and E. Latz, Nat. Rev. Immunol., 2010, 10, 123-130.

161 Y. Tanaka and Z. J. Chen, Sci. Signaling, 2012, 5, ra20.

162 T. Abe, A. Harashima, T. Xia, H. Konno, K. Konno, A. Morales, J. Ahn, D. Gutman and G. N. Barber, Mol. Cell, 2013, 50, 5-15.

163 D. L. Burdette, K. M. Monroe, K. Sotelo-Troha, J. S. Iwig, B. Eckert, M. Hyodo, Y. Hayakawa and R. E. Vance, Nature, 2011, 478, 515-518.
164 D. K. Karaolis, M. H. Rashid, R. Chythanya, W. Luo, M. Hyodo and Y. Hayakawa, Antimicrob. Agents Chemother., 2005, 49, 1029-1038.

165 D. K. R. Karaolis, T. K. Means, D. Yang, M. Takahashi, T. Yoshimura, E. Muraille, D. Philpott, J. T. Schroeder, M. Hyodo, Y. Hayakawa, B. G. Talbot, E. Brouillette and F. Malouin, J. Immunol., 2007, 178, 2171-2181.

166 D. K. R. Karaolis, K. R. Cheng, M. Lipsky, A. Elnabawi, J. Catalano, M. Hyodo, Y. Hayakawa and J. P. Raufman, Biochem. Biophys. Res. Commun., 2005, 329, 40-45.

167 D. Chandra, W. Quispe-Tintaya, A. Jahangir, D. Asafu-Adjei, I. Ramos, H. O. Sintim, J. Zhou, Y. Hayakawa, D. K. Karaolis and C. Gravekamp, Cancer Immunol. Res., 2014, 2, 901-910.

168 S. Yildiz, E. Alpdundar, B. Gungor, T. Kahraman, B. Bayyurt, I. Gursel and M. Gursel, Eur. J. Immunol., 2015, 45, 1170-1179.

169 B. J. Koestler, S. S. Seregin, D. P. Rastall, Y. A. Aldhamen, S. Godbehere, A. Amalfitano and C. M. Waters, Clin. Vaccine Immunol., 2014, 21, 1550-1559.

170 F. S. Alyaqoub, Y. A. Aldhamen, B. J. Koestler, E. L. Bruger, S. S. Seregin, C. Pereira-Hicks, S. Godbehere, C. M. Waters and A. Amalfitano, J. Immunol., 2016, 196, 1741-1752.

171 H. Miyabe, M. Hyodo, T. Nakamura, Y. Sato, Y. Hayakawa and H. Harashima, J. Controlled Release, 2014, 184, 20-27.

172 T. Nakamura, H. Miyabe, M. Hyodo, Y. Sato, Y. Hayakawa and H. Harashima, J. Controlled Release, 2015, 216, 149-157.

173 X. D. Li, J. Wu, D. Gao, H. Wang, L. Sun and Z. J. Chen, Science, 2013, 341, 1390-1394. 